\title{
ÉTUDE EXPLORATOIRE DU RAISONNEMENT CLINIQUE CHEZ LES ÉTUDIANTS EN SOINS INFIRMIERS À L'AIDE DE LA CARTE CONCEPTUELLE
}

Exploratory study of clinical reasoning in nursing students with the conceptual map

\section{Sylvie PAUCARD-DUPONT, \\ Cadre de santé, IFSI Paris}

Master professionnel de Santé publique. Spécialité : Ingénierie des formations en santé.

Université Paris 13 Sorbonne-Paris-Cité

\author{
Claire MARCHAND, \\ Maître de Conférences \\ Laboratoire Educations et Pratiques de Santé EA 3412 \\ Université Paris 13 Sorbonne-Paris-Cité
}

Adresse électronique : sylvie.dupont30@wanadoo.fr

Adresse postale : 38 , rue Letellier 75015 Paris

Numéro de téléphone : 0667318590 


\section{RÉSUMÉ}

Contexte : Le référentiel de formation conduisant au diplôme d'État d'Infirmier(e) place l'apprentissage du raisonnement clinique au cœur du dispositif de formation. Nous nous sommes interrogés sur les possibilités de rendre visible les processus mentaux qui s'opèrent chez les étudiants en soins infirmiers quand ils raisonnent afin de repérer leurs stratégies et leurs difficultés de raisonnement. Il s'avère que la carte conceptuelle constitue un outil de recherche capable de montrer ces deux aspects.

Objectifs : Le but de ce travail est de vérifier qu'une carte conceptuelle réalisée au cours d'un entretien et construite à partir du discours d'un étudiant en soins infirmiers lorsqu'il analyse une situation clinique simulée, est en mesure de rendre visible ses stratégies de raisonnement clinique et ses difficultés de raisonnement. Dans un second temps, il s'agit d'explorer à quel point la carte conceptuelle une fois élaborée permet aux étudiants d'identifier leur propre fonctionnement intellectuel.

Méthode : 12 étudiants en soins infirmiers de $2^{\text {ème }}$ année ont participé à l'étude. Les cartes conceptuelles ont été construites par le formateur/chercheur au fur et à mesure que les étudiants analysaient à voix haute une situation clinique simulée écrite. Les cartes ont été analysées à partir d'une grille de référence.

Des entretiens ont été menés à la suite de l'élaboration des cartes conceptuelles et les commentaires des étudiants ont été analysés.

Résultats: Les étudiants ont utilisé des stratégies de raisonnement mixtes avec une dominante inductive $(5 / 12)$ ou une dominante hypothético-déductive (5/12). Les difficultés de raisonnement identifiées sont liées à l'absence de repérage d'informations importantes, à l'absence d'analyse des données, à l'absence de liens ou l'existence de liens erronés. L'analyse des commentaires a mis en évidence que la carte conceptuelle contribuait au développement d'habiletés métacognitives.

Conclusion : La carte conceptuelle présente un intérêt pour contribuer à une évaluation diagnostique des apprentissages du raisonnement clinique. Elle constitue une aide au développement des habiletés métacognitives chez les étudiants. Cet outil peut s'avérer utile pour mettre en œuvre des stratégies d'aide à l'apprentissage au raisonnement clinique.

Mots clés: Formation en soins infirmiers, stratégies de raisonnement clinique, carte conceptuelle, habiletés métacognitives.

\section{ABSTRACT}

Background: The training reference leading to the state nursing diploma places the learning of clinical reasoning at the center of the training. We have been wondering about the possibilities of making visible the student nurse's mental processes when they provide nursing care in order to identify their strategies and reasoning difficulties. It turns out that the concept map is a research tool capable of showing these two aspects.

Objectives: The aim of this study is to verify a conceptual map made during an interview and built from the speech of a nursing student when analyzing a simulated clinical situation, is able to make visible its strategies clinical reasoning and reasoning difficulties. In a second phase of it, is to explore how the concept map once elaborated allows students to identify their own intellectual reasoning.

Method: 12 nursing second year students have participated in the study. Concept maps were constructed by the trainer / researcher as the students analyzed aloud a simulated clinical situation written. Concept maps were analyzed from a reference grid. Interviews were conducted following the elaboration of concept maps and student's comments were analyzed. Results: Students reasoning strategies were either mixed inductive dominant (5/12) or hypothetical-deductive dominant (5/12). Reasoning difficulties identified are related to the lack of identification of important information, the lack of analysis of data, lack of connection 
or the existence of faulty links. Analysis of the comments highlights that conceptual map contributed to the development of metacognitive skills.

Conclusion: The concept map has shown benefits in contributing to a diagnostic assessment of clinical reasoning learning. It is an additional resource tool to facilitate the development of metacognitive skills for students. This tool can be useful to implement support learning strategies in clinical reasoning.

Key words: Training of nursing care, clinical reasoning strategies, concept mapping, metacognitive skills. 


\section{REMERCIEMENTS:}

L'auteur remercie Claire Marchand pour ses conseils et son accompagnement tout au long de ce travail de Master en Santé Publique, spécialité Ingénierie des Formations en Santé, ainsi que l'ensemble des étudiants en soins infirmiers pour leur participation à l'étude. 


\section{INTRODUCTION}

Le référentiel du diplôme d'État d'Infirmier(e) s'articule autour de dix compétences. La compétence $1:$ «Évaluer une situation clinique et établir un diagnostic dans le domaine infirmier» et la compétence 2 : «Concevoir et conduire un projet de soins infirmiers » mettent en évidence une vision du métier d'infirmier basée sur l'évaluation de situation clinique (Ministère des Affaires Sociales et le la Santé, 2009) [1]. Transversales, ces deux compétences sont en relation directe avec les huit autres. Le référentiel de compétences place ainsi l'infirmier dans une pratique réflexive basée sur le raisonnement clinique, (Coudray, 2009) [2]. L'apprentissage du raisonnement clinique est donc au cœur du dispositif de formation. Ce paramètre engage le formateur à développer des méthodes pédagogiques qui mobilisent les opérations mentales du raisonnement clinique : induction, déduction, hypothético-déduction, mais aussi il l'incite à mener une réflexion pédagogique sur ce sujet. Comment rendre visible les processus mentaux qui s'opèrent chez l'étudiant quand il raisonne ? Comment repérer les stratégies de raisonnement mises en œuvre par les étudiants ? Comment détecter les dysfonctionnements cognitifs pour mieux accompagner les étudiants dans cet apprentissage et élaborer des stratégies de remédiation? La carte conceptuelle pourrait être un outil de recherche capable de fournir des données utiles et valides sur la démarche mentale qui s'opère quand les étudiants en soins infirmiers raisonnent.

\section{Le raisonnement clinique}

L'acte de raisonner consiste en « une activité mentale qui permet de produire par inférence des informations nouvelles à partir d'informations qui ne la contiennent pas explicitement...» (Raynal, Rieunier, 2010) [3]. C'est donc un processus intellectuel invisible, la personne qui raisonne effectue une série d'inférences et le résultat de son raisonnement se traduit le plus souvent par une action. Dans le domaine de la santé, le raisonnement clinique désigne le processus de pensée et de prise de décision en regard d'un problème de santé. Cependant, ce raisonnement est très souvent implicite et sa formalisation écrite ne reproduit pas toujours l'ampleur de la démarche intellectuelle qui s'opère. C'est à partir des données recueillies auprès du patient que l'étudiant en soins infirmier active des opérations mentales. C'est ainsi qu'il relie des signes à un problème de santé. Il est aussi en mesure de raisonner par anticipation, c'est-à-dire qu'il émet un problème de santé qu'il vérifie ensuite en le confrontant à un recueil de données cliniques auprès du patient (Psiuk, 2012) [4].

L'étude du raisonnement clinique existe depuis de nombreuses années. Il est considéré comme un processus de pensée et de prise de décision hautement complexe. Nendaz, Charlin, Leblanc et Bordage, 2005 [5], en ont proposé une synthèse afin d'aider les enseignants en santé à mieux l'enseigner et l'évaluer auprès de leurs étudiants. Deux types de processus sont habituellement distingués tout en pouvant être activés ensemble lors de la résolution d'un problème : les processus analytiques et les processus non analytiques. Les raisonnements non analytiques relèvent d'une pensée intuitive, réflexe ou automatique s'appuyant le plus souvent sur la reconnaissance d'une similitude entre le problème posé et les expériences antérieures du professionnel. C'est un processus qui se situe au dessous du seuil perceptible de la conscience. Les raisonnements non analytiques sont surtout retrouvés chez les professionnels expérimentés, ayant stockés en mémoire à long terme de nombreux cas et raisonnements. C'est un processus de raisonnement qui se développe avec l'expérience clinique. De fait, il est probable que ce type de raisonnement soit encore peu présent chez des étudiants en soins infirmiers (en dehors de ceux qui auraient une expérience clinique préalable). Ils utiliseraient plutôt des modes de raisonnement analytique de type hypothéticodéductif ou inductif. Lors d'un raisonnement hypothéticodéductif considéré aujourd'hui comme un phénomène irrépressible, l'étudiant émet quelques hypothèses diagnostiques précocement. Il cherche 
ensuite à les confirmer ou à les infirmer au travers d'un recueil d'informations complémentaires validant ou infirmant progressivement ses premières hypothèses, puis en en émettant de nouvelles si besoin (Eva, 2005) [6]. C'est un mode de raisonnement qui va de la solution à l'information. L'autre mode de raisonnement utilisable par les étudiants est celui qui consiste à cheminer des données vers la solution (Masquelet, 2006) [7]. Dans ce cas, l'étudiant partirait des données qu'il a en sa possession et appliquerait des règles et principes stockés en mémoire pour aboutir au diagnostic. Il s'agirait d'un mode de raisonnement plus inductif utiliser plus spécifiquement lorsque l'étudiant ou le professionnel ne perçoit pas d'emblée d'hypothèse pertinente devant un tableau clinique complexe.

Les processus de raisonnement clinique infirmier se fondent autour de trois domaines cliniques : le premier concerne les signes et symptômes de la maladie, le second rassemble l'ensemble des risques liés à la pathologie, le troisième fait référence aux réactions humaines physiques et psychologiques du patient face à ses problèmes de santé. Ainsi, le jugement clinique qu'opère l'infirmier est la résultante d'un recueil de données qui a permis d'identifier la présence de signes importants. Il s'agit d'un modèle clinique tri focal qui favorise « une vision holistique de la situation clinique d'un patient » (Psiuk, 2012) [4].

C'est ainsi que Psiuk, 2012 [4] définit le raisonnement clinique comme : "une démarche systématique qui intègre et met en lien, à partir d'un examen physique et d'une écoute active, les signes et les symptômes recueillis, permet l'élaboration d'hypothèses de problèmes de santé réels ou potentiels et d'hypothèses de réactions humaines physiques et comportementales réelles ou potentielles, cette démarche se terminent par la validation de l'hypothèse la plus probable »

Nous retrouvons dans la littérature plusieurs travaux de recherche en éducation médicale qui se sont penchés sur l'étude des processus qui caractérisent le raisonnement clinique afin d'aider les enseignants cliniciens à mieux accompagner son apprentissage (Nendaz, Charlin, Leblanc et Bordage, 2005) [5] ; (Eva, 2005) [6] ; (Audétat, Laurin, Sanche, 2011) [8]. Peu d'études en revanche ont exploré les mécanismes de raisonnement clinique mise en œuvre par les étudiants en soins infirmiers en phase d'apprentissage.

\section{La psychologie cognitive}

Les théories cognitivistes de l'apprentissage préconisent de s'intéresser à la manière dont un étudiant construit ses connaissances et à comprendre comment il les organise dans sa structure mentale (Tardif, 1997) [9]. Elles précisent aussi qu'un apprentissage porteur de sens est obligatoirement lié, entre autre, à l'organisation des connaissances en mémoire. Des études ont montrées que plus les connaissances sont organisées et sont reliées entre elles sous forme de réseaux dans la mémoire à long terme de l'apprenant, plus son raisonnement clinique est performant et plus il est en mesure d'associer de nouvelles informations qu'il peut réutiliser fonctionnellement (Tardif, 1997) [9] ; (Demeester, Vanpee, Marchand, Eymard, 2010) [10]. L'intérêt de pouvoir identifier dans quelle mesure les connaissances sont organisées, mises en lien et en réseaux est déterminant pour la mise en œuvre de stratégies d'enseignement adaptées aux besoins des étudiants.

La carte conceptuelle comme outil d'exploration du raisonnement clinique

C'est Novak qui a développé la cartographie conceptuelle. Elle trouve son origine au sein des recherches menées par Ausubel en psychologie cognitive (Tardif, 2006) [11]. Vitale et Romance cité par Tardif, 2006, [11] caractérisent la carte conceptuelle comme un puissant outil qui permet d'identifier les connaissances qu'utilisent les apprenants dans le cadre de 
résolution de problèmes complexes. Cet outil permet de représenter graphiquement les connaissances elles-mêmes et les relations entre ces connaissances.

Demeester, Vanpee, Marchand, Eymard 2010, [10] expliquent que « la construction de carte conceptuelle est utilisée en tant que stratégie de représentation des connaissances ».

En effet, l'élaboration d'une carte conceptuelle permet de rendre visible la pensée d'une personne à travers l'organisation des concepts qu'elle y fait figurer (Marchand, d'Ivernois, 2004) [12]. En construire une consiste donc à créer une représentation graphique, qui prend la forme d'un réseau élaboré à partir d'un ensemble de concepts reliés entre eux par une flèche et une expression verbale précisant le sens de la relation qui au final traduit une connaissance (Demeester, Vanpee, Marchand, Eymard, 2010) [10] ; (Novak, Canas, 2008) [13].

Une analyse de la littérature portant sur l'usage des cartes conceptuelles en pédagogie médicale a souligné les nombreux avantages de leur utilisation (Daley, 2010) [14]. En particulier, la carte conceptuelle permet d'explorer l'organisation et l'utilisation des connaissances, mais aussi d'identifier des lacunes, des connaissances erronées ou des difficultés de mise en lien entre les concepts (Marchand, d'Ivernois, 2004) [12]. Dans le cadre spécifique de l'évaluation du raisonnement clinique la carte conceptuelle aurait plusieurs propriétés : celle de visualiser comment l'apprentissage se réalise, celle d'apprécier la façon dont l'apprenant construit et organise ses connaissances, celle de mettre en évidence les processus de pensée, celle de représenter et d'exprimer des habiletés intellectuelles. Ainsi, cet outil répondrait d'avantage à des critères centrés sur l'évaluation formative ou diagnostique (Demeester, Vanpee, Marchand, Eymard, 2010) [10]. Il convient également de rajouter que l'élaboration d'une carte conceptuelle est une stratégie intéressante pour le développement des capacités métacognitives de l'apprenant (Marchand, Gagnayre, 2004) [15].

Au regard de l'ensemble de ces atouts, la carte conceptuelle semble être un outil d'évaluation intéressant pour le formateur, étant donné que peu d'instruments d'évaluation sont en mesure de déterminer la façon dont un apprenant construit et organise ses connaissances (Marchand, d'Ivernois, 2004) [12].

Pottier, Planchon, Hardouin, Sebille, Rogez, Barrier, 2011, [16] ont voulu montrer la validité d'un dispositif cartographique pour l'étude du raisonnement clinique. L'objectif de leur recherche était d'explorer la viabilité de ce dispositif pour rendre compte de la structure des processus de raisonnement clinique développés en situation expérimentale chez les étudiants en médecine. Cette étude fait apparaître que la représentation du raisonnement clinique montrée par les cartes retrouve les différentes stratégies de résolution de problèmes rapportées dans la littérature. Par ailleurs, les auteurs suggèrent que cet outil d'évaluation des processus mentaux pourrait être utilisé pour repérer les étudiants en difficulté d'apprentissage. Audétat, Laurin, Sanche, 2011, [8] mettent en avant que reconnaitre précocement les difficultés de raisonnement clinique d'un apprenant peut permettre la mise en œuvre d'un plan pédagogique individualisé assez tôt dans la formation, de façon à soutenir sa progression vers le degré de maîtrise des compétences attendues pour son niveau de formation.

Sachant que les prouesses du raisonnement clinique dépendent de la qualité de l'organisation des connaissances dans la mémoire à long terme et de la manière dont ces connaissances sont reliées entre elles sous formes de réseaux (Demeester, Vanpee, Marchand, Eymard, 2010) [10], observer ces processus par l'intermédiaire de la carte conceptuelle semble présenter un intérêt pédagogique indéniable. Pour identifier le processus mental qui s'opère quand les étudiants en soins infirmiers raisonnent, une des possibilités est que la carte conceptuelle soit élaborée par une tierce personne au cours d'un entretien à partir du discours de l'étudiant (Pottier, Planchon, Hardouin, Sebille, Rogez, Barrier 2011) [16] ; (Marchand, d'Ivernois, Assal, Slama, Hivon, 2002)[17]. 
Ainsi l'objectif principal de cette étude exploratoire cherchera à vérifier qu'une carte conceptuelle réalisée au cours d'entretiens et construite à partir du discours des étudiants en soins infirmiers lorsqu'ils analysent une situation clinique simulée, est en mesure de rendre visible leurs stratégies de raisonnement clinique et leurs difficultés de raisonnement.

L'objectif secondaire est d'explorer à quel point la carte conceptuelle une fois élaborée permet aux étudiants d'identifier leur propre fonctionnement intellectuel.

\section{MÉTHODES}

Cadre de l'étude

L'expérimentation a lieu au cours du $4^{\text {ème }}$ semestre de la formation, auprès d'une douzaine d'étudiants en soins infirmiers post-novices. Ces étudiants ont suivi les enseignements rattachés au raisonnement, à la démarche clinique infirmière et au projet de soins infirmiers avec des études de cas simulés. Ils ont réalisés un stage de cinq semaines et deux stages de dix semaines dans quatre types de stages représentatifs de «familles de situations » (Soins de courte durée, soins en santé mentale et en psychiatrie, soins de longue durée et soins de suite et réadaptation, soins individuels ou collectifs sur des lieux de vie).

Nous avons fait le choix de proposer l'expérience aux étudiants de notre groupe de suivi pédagogique, 18 au total. Dans un premier temps, nous avons expliqué le but et le déroulement de notre recherche. Nous avons ensuite demandé qui parmi le groupe d'étudiants souhaitait se porter volontaire pour participer à l'étude; 12 d'entre eux se sont portés volontaires.

Nous avons construit une situation clinique simulée réaliste et complexe (Annexe I) que les étudiants ont analysée individuellement pendant les entretiens.

\section{Description de la population}

L'échantillon constitué de 12 étudiants en soins infirmiers rassemble 9 étudiantes de sexe féminin et 3 étudiants de sexe masculin. Il est hétérogène par l'âge, l'ethnie, le niveau de formation et l'expérience professionnelle. L'âge moyen est de 32 ans et demi, le profil du groupe est représentatif de la promotion complète. Le plus jeune à 21 ans, le plus âgé à 45 ans. Six étudiants sont titulaires du baccalauréat, sept sont titulaires du diplôme d'état d'aidesoignant, deux sont titulaires d'un diplôme universitaire.

\section{Choix des outils de recherche et déroulement de l'étude}

L'étude exploratoire s'est déroulée sur six jours entre le mois de mars et avril 2013 à raison de deux entretiens par jour. Des entretiens utilisant des techniques d'aide à l'explicitation (Vermersch, 2000) [18] ont été menés avec élaboration d'une carte conceptuelle au cours des entretiens avec les étudiants. La contribution des techniques d'aide à l'explicitation pour construire la carte conceptuelle a permis d'aider l'étudiant à verbaliser son action de raisonner. Les questions que nous avons utilisées pendant l'entretien ont contribué à recentrer le discours de l'étudiant sur son action d'analyse de la situation clinique, à lui faire expliciter sa démarche mentale, à lui faire justifier son questionnement et ses choix.

Les cartes ont été construites par nous même à partir du raisonnement exprimé à voix haute par l'étudiant. La situation clinique simulée a été soumise à chaque étudiant en début d'entretien. Un temps de lecture du cas leur a été accordé avant qu'ils expriment à voix haute leur raisonnement.

La technique du raisonnement à voix haute a été retenue pour que l'étudiant nous fasse part du cheminement intellectuel qu'il emprunte lorsqu'il raisonne à partir d'une situation clinique (Phaneuf, 2009) [19]. Par ailleurs nous avons souhaité représenter nous-même graphiquement 
et simultanément, sous forme de cartes conceptuelles la structure cognitive des étudiants à partir de leur discours (Marchand, d'Ivernois, Assal, Slama, Hivon, 2002) [17] car d'après Pottier, Planchon, Hardouin, Sebille, Rogez, Barrier, 2011, [16] raisonner et construire une carte en même temps pouvait être un exercice difficile pour les étudiants.

En début de séance les consignes suivantes ont été formulées à chaque étudiant : «Après la lecture de la situation clinique, vous exprimerez à haute voix le raisonnement que vous adoptez pour analyser cette situation. De mon côté, je dessinerai sous forme de schéma votre raisonnement. Au cours de votre discours, je serai peut-être amenée à vous interrompre régulièrement, pour vous demander de clarifier votre raisonnement et valider au fur et à mesure avec vous si je reproduis correctement le processus de votre pensée. A la fin de l'entretien je vous demanderai si vous avez des choses à rajouter, à compléter à la vue de la carte graphique que j'aurai dessinée. Ensuite je vous demanderai de regarder la carte et de me dire le regard que vous posez sur votre démarche mentale quand vous analysez une situation clinique».

En fin d'entretien le choix de demander à l'étudiant le regard qu'il pose sur sa démarche mentale repose sur l'idée d'identifier si à la lecture de la carte, l'étudiant prend conscience de son propre fonctionnement intellectuel.

Aucune contrainte de temps n'a été imposée aux étudiants. Chaque entretien a duré en moyenne 48 minutes. L'accord de chaque participant a été obtenu pour l'enregistrement audio des entretiens.

\section{Modalités d'analyse}

\section{Analyse des cartes}

L'analyse des 12 cartes conceptuelles construites au cours des entretiens a suivi un protocole qualitatif.

Le contenu des enregistrements a été réécouté et les cartes ont été retranscrites en utilisant le logiciel «Cmap tools» pour être ensuite analysées. Dans un premier temps nous avons cherché à déterminer les stratégies de raisonnement clinique utilisées par les étudiants ; l'idée étant de définir des tendances de raisonnement à dominante inductive ou hypothéticodéductive. Pour cela, nous nous sommes appuyées sur la nature des liens retrouvés dans les cartes. Nous avons différencié les liens du type causal ou conditionnel (Nendaz, Charlin, Leblanc et Bordage, 2005) [5] (ex : c'est dû, si....alors) pour déterminer le raisonnement inductif et les liens d'effet (ex: peut expliquer, caractérisé par) pour déterminer le raisonnement hypothético déductif. Il convient de souligner que nous avons aussi pris en compte le sens des flèches pour déterminer le modèle de raisonnement inductif ou hypothético déductif. En effet, si les flèches allaient des faits vers les hypothèses nous avons déduit qu'il s'agissait d'un raisonnement inductif, inversement si les flèches allaient des hypothèses vers les faits nous avons déduit qu'il s'agissait d'un raisonnement hypothéticodéductif. La nature des liens retrouvés dans les cartes a été recensée dans une grille d'analyse.

Dans un deuxième temps, nous avons cherché à identifier les difficultés de raisonnement chez les étudiants. Pour cela, nous avons confronté les cartes conceptuelles à une grille de référence (Annexe II) élaborée par nous-même.

Nous avons classé les difficultés en trois catégories.

- 1. Difficulté à l'identification des problèmes.

Nous avons cherché ici à repérer si les étudiants avaient identifié l'ensemble ou une partie des problèmes de santé que présentait le patient ainsi que le problème social.

- 2. Difficulté à identifier les trois domaines cliniques (Psiuk, 2012) [4].

Nous avons noté ici si les étudiants prenaient en compte l'ensemble ou une partie : des signes et symptômes relatifs aux pathologies que présentaient le patient (premier 
domaine); des risques liés aux pathologies, aux effets secondaires des traitements, aux contextes intrinsèques et extrinsèques (deuxième domaine); des réactions humaines physiques et/ou psychologiques du patient (troisième domaine).

- 3. Difficulté à établir les liens entre différents problèmes (ou éléments) :

Nous avons exploré si des liens existaient entre différents problèmes (lien présent ou absent) ainsi que leur validité (lien juste ou erroné) : Lien entre problèmes de santé et risques, liens entre les problèmes de santé et les réactions humaines physiques et/ou psychologiques, liens entre les réactions humaines physiques et/ou psychologiques, liens entre les réactions humaines physiques et/ou psychologiques et les risques, liens entre les signes et les problèmes de santé, liens entre les données observées et les réactions physiques et/ou psychologiques.

Il convient de préciser que la hiérarchisation n'a pas pu être prise en compte dans l'analyse étant donné que les cartes ont été construites au fur et à mesure que l'étudiant a exprimé à voix haute son raisonnement.

Afin de pouvoir repérer et recenser plus facilement les problèmes de santé, le problème social, les données, les signes et symptômes, les risques, les réactions humaines physiques et/ou psychologiques, ainsi que les liens sur les cartes conceptuelles, nous avons utilisé des codes couleurs (figure 1).

Analyse des réponses à la question sur la démarche mentale

Les réponses produites par les étudiants à partir d'une question sur leur démarche mentale ont fait l'objet d'une analyse de contenu (Fortin, 2010) [20].

Six catégories d'analyse, issues des réponses obtenues ont été définies :

- $\quad$ 1. Les jugements spontanés que portent les étudiants sur leur démarche mentale. .

- 2. Les habiletés métacognitives. Nous avons classé ici les éléments des verbatim des entretiens témoignant d'une capacité de l'étudiant à analyser son propre fonctionnement intellectuel quand il raisonne.

- 3. Les intérêts que les étudiants attribuent à la carte conceptuelle.

- 4. Les difficultés de verbalisation par les étudiants à propos de leur démarche mentale. Nous avons relevé ici les propos, les silences, témoignant de difficultés à dire, à expliciter son action, son raisonnement, ainsi que les discours témoignant des difficultés à dire tout simplement ce que la carte conceptuelle leur renvoie.

- 5. Les interrogations que suscite l'exercice chez les étudiants.

- 6. Autres.

Les verbatim des étudiants ont été découpés en unités de sens et classés au sein de ces catégories quand ils s'y rapportaient. 


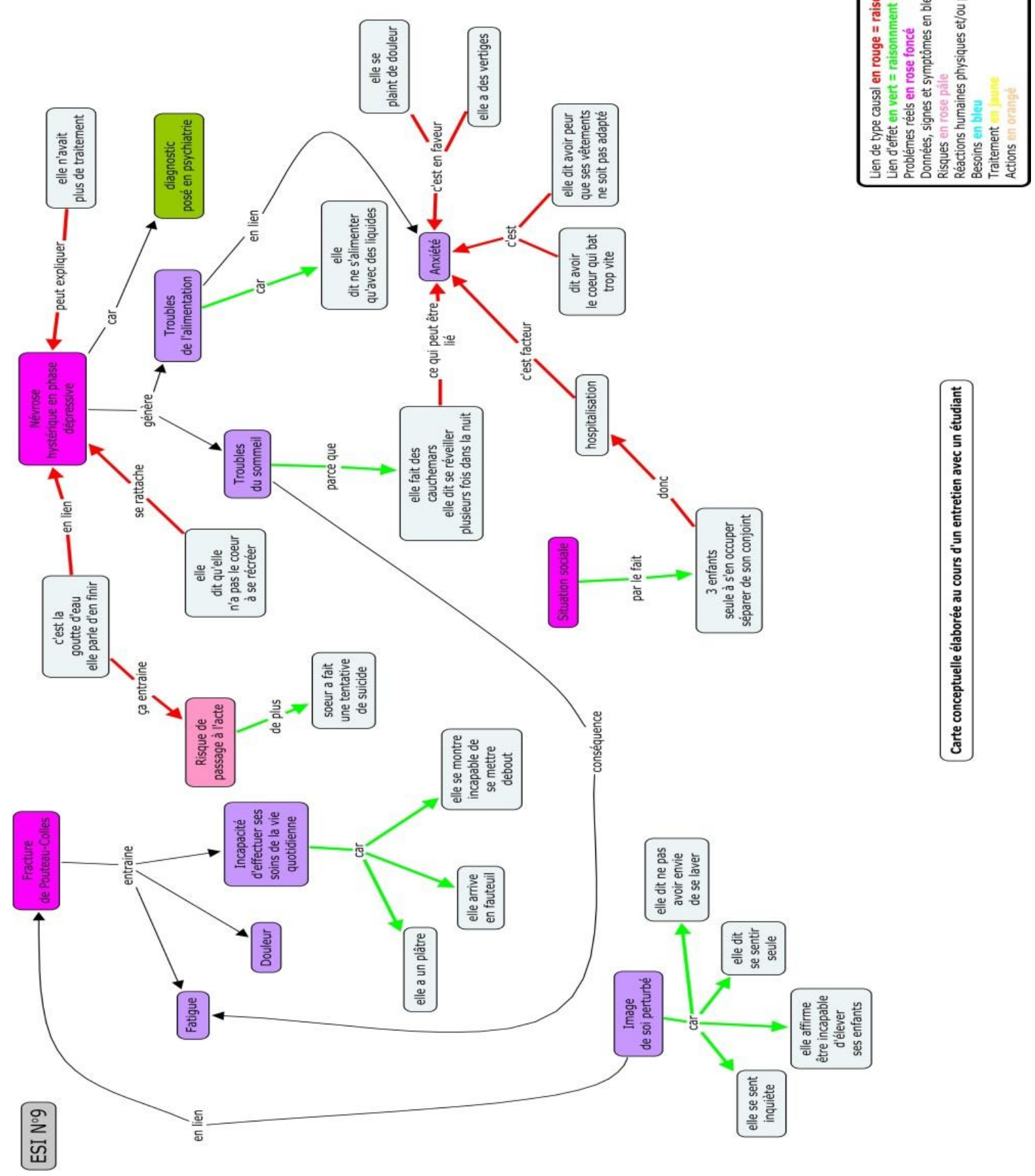

Figure 1 : Carte conceptuelle (exemple) 


\section{RESULTATS}

12 cartes conceptuelles (Annexe III), ont été élaborées à partir du discours des étudiants $(n=12)$ et analysées. Le tableau 1 propose une synthèse des éléments d'analyse des cartes conceptuelles.

\section{Stratégie de raisonnement}

Dans un premier temps nous avons relevé les stratégies de raisonnement qu'utilisent les étudiants lorsqu'ils analysent une situation clinique simulée. La plupart des étudiants (10/12) utilisent des stratégies mixtes mais néanmoins avec des dominantes : 5 étudiants raisonnent avec une dominante plutôt inductive et 5 raisonnent avec une dominante hypothéticodéductive. Deux autres étudiants ne présentent pas de dominante, ils raisonnent selon un modèle analytique entièrement mixte. Aucun étudiant n'opère un raisonnement exclusivement inductif ou exclusivement hypothético-déductif.

\section{Difficultés de raisonnement}

Identification des problèmes de santé

La moitié des étudiants (6/12) ont identifié l'ensemble des problèmes de santé et le problème social (3 problèmes sur 3) présent dans la situation clinique simulée. Tous les étudiants $(\mathrm{n}=12)$ ont au moins retrouvé un problème de santé parmi les 3 attendus. Les problèmes de santé les plus retrouvés sont :

- Le problème psychiatrique $(11 / 12)$

- Le problème orthopédique (10/12)

- Le problème social (7/12).

Trois étudiants ont évoqué un problème cardiaque et 1 a évoqué un problème neurologique. Ces 2 problèmes n'étaient pas attendus dans la mesure où il manque des données dans le texte qui permettent d'affirmer ces deux hypothèses.

\section{Identification des domaines cliniques}

- Les données, les signes et les symptômes

Un quart des étudiants (3/12) ont pris en compte l'ensemble des données, signes et symptômes (environ 17 attendus), plus de la moitié (8/12) en a identifié la plupart. Un seul étudiant n'en a relevé que quelques-uns et énonce essentiellement ce qu'il n'identifie pas dans la situation simulée : «je ne vois pas si elle mange bien » «on ne dit pas si elle est constipée, si elle a la diarrhée $»$.

\section{- Les risques}

Aucun étudiant n'a identifié l'ensemble des risques (5 risques attendus) liés aux pathologies, aux effets secondaires ou au contexte intrinsèques et extrinsèque. Plus de la moitié des étudiants (8/12) ont au moins identifié un risque sur cinq. Le risque de tentative de suicide par exemple a été évoqué par 7 étudiants. Le risque infectieux, le risque de syndrome de Volkmann, le risque de dénutrition n'ont été évoqués par aucun étudiant. Quatre étudiants n'ont identifié aucuns risques.

- Les réactions humaines physiques et/ou psychologiques

Un seul étudiant énonce l'ensemble des réactions humaines du patient face à ses problèmes de santé et son problème social (9 éléments attendus). Sept étudiants en énoncent la plupart soit entre 6 et 8 réactions. Un seul étudiant n'énonce aucunes réactions humaines. Ce dernier répète la liste des besoins fondamentaux présentés dans la situation simulée : «pour être propre et soigner ses téguments » « se mouvoir et maintenir une bonne posture ».

Les réactions humaines physiques et/ou psychologiques les plus citées sont : 
- L'anxiété (10/12)

- $\quad$ La douleur (10/12)

- Perturbation des habitudes du sommeil (7/12)

- Incapacité à effectuer les gestes de la vie quotidienne (6/12).

\section{Les liens de sens établis entre les différents éléments de la carte}

- Les liens établis entre les problèmes de santé et les risques

La moitié des étudiants (6/12) ont établis des liens justes entre problème de santé et risques. Deux n'ont pas établis de liens. Par exemple, le risque de chute n'est pas mis en lien avec le problème psychiatrique. Pour les autres (4/12) nous ne sommes pas en mesure d'évaluer leur capacité à établir les liens entre problèmes de santé et risques car ils n'ont pas identifié de risques.

- Les liens établis entre problèmes de santé et réactions humaines

Pour la majorité des étudiants la présence de liens est repérée (11/12). Mais pour 8 d'entre eux des liens possibles entre problèmes de santé et réactions humaines sont absents. Par exemple l'anxiété n'est pas mise en lien avec le problème psychiatrique, ni avec le problème social; l'altération de la mobilité physique n'est pas mise en lien avec le problème orthopédique et le problème psychiatrique; la douleur n'est pas mise en lien avec le problème orthopédique; la perturbation des habitudes de sommeil n'est pas mis en lien avec le problème social et/ou le problème psychiatrique.

La plupart des liens présents entre problèmes de santé et réactions humaines sont justes, sauf trois qui sont erronés. Trois étudiants sont concernés. Ceux-ci établissent des liens alors qu'il manque des éléments dans le texte pour les objectiver.

- Les liens établis entre les réactions humaines physiques et/ou psychologiques entre elles.

Plus de la moitié des étudiants (7/12) ont établis des liens entre les réactions humaines ; pour l'ensemble, les liens sont justes.

- Les liens entre les réactions humaines physiques et/ou psychologiques et les risques Un quart des étudiants ont créé des liens entre les réactions humaines et les risques. Ces liens sont justes.

- Les liens établis entre les signes et les problèmes de santé réels

Quand les étudiants (10/12) établissent des liens entre les signes et les problèmes de santé, ces liens sont justes. Néanmoins pour certains étudiants (5/12), des signes significatifs du problème psychiatrique ne sont pas rattachés à ce problème. Par exemple les signes suivants : visage exprime de la lassitude, paupières baissées, propos réduit, débit lent, ces signes ne sont pas mis en lien avec le problème psychiatrique.

- Les liens établis entre les données observées et les réactions humaines

La majorité des étudiants (11/12) établissent des liens justes entre les données et les réactions humaines. Cependant 3 d'entre eux établissent des liens erronés. Par exemple les données suivantes :

- «il vaudrait mieux en finir » est rattachée à l'anxiété et non au risque de suicide.

- «elle a peur que ses vêtements ne soient pas adaptés à son plâtre » est rattachée à un déficit en auto-soins et non à l'anxiété. 
Pour un certain nombre d'étudiants, notons que nous n'avons pas toujours pu évaluer leur capacité à faire les liens dans la mesure où ils n'ont identifié ni risques, ni réactions humaines ou du moins pas de façon explicite.

Tableau 1 : Synthèse des résultats

\begin{tabular}{|c|c|c|c|c|c|c|c|c|c|c|c|}
\hline & \multirow[b]{3}{*}{$\begin{array}{l}\text { Stratégie de } \\
\text { raisonnement }\end{array}$} & \multicolumn{10}{|c|}{ Identification des difficultés de raisonnement } \\
\hline & & \multirow[b]{2}{*}{ 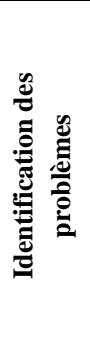 } & \multicolumn{3}{|c|}{$\begin{array}{c}\text { Identification des trois domaines } \\
\text { cliniques }\end{array}$} & \multicolumn{6}{|c|}{ Les liens établis } \\
\hline & & & 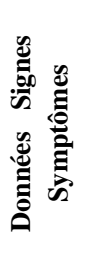 & 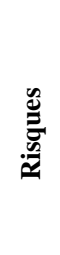 & 总 & 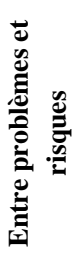 & 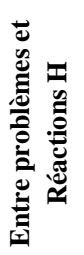 & 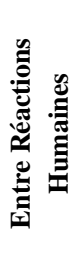 & 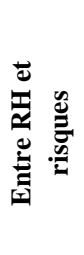 & 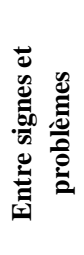 & 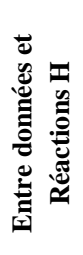 \\
\hline ESI 1 & $\begin{array}{c}\text { Mixte } \\
\text { Dominante } \\
\text { hypothético-déductif }\end{array}$ & $3 / 3$ & $17 / 17$ & $2 / 5$ & $7 / 9$ & abst & $\begin{array}{c}\text { justes } \\
+ \\
\text { abst }\end{array}$ & justes & justes & $\begin{array}{c}\text { justes } \\
+ \\
\text { abst }\end{array}$ & $\begin{array}{c}\text { justes } \\
+ \\
\text { erroné }\end{array}$ \\
\hline ESI 2 & Mixte & $3 / 3$ & $10 / 17$ & $2 / 5$ & $3 / 9$ & justes & $\begin{array}{c}\text { justes } \\
+ \\
\text { abst }\end{array}$ & justes & justes & justes & justes \\
\hline ESI 3 & $\begin{array}{c}\text { Mixte } \\
\text { Dominante } \\
\text { inductif }\end{array}$ & $3 / 3$ & $17 / 17$ & $0 / 5$ & $3 / 9$ & & justes & & & justes & justes \\
\hline ESI 4 & $\begin{array}{c}\text { Mixte } \\
\text { Dominante } \\
\text { inductif } \\
\end{array}$ & $2 / 3$ & $4 / 17$ & $1 / 5$ & $0 / 9$ & abst & & & & justes & \\
\hline ESI 5 & $\begin{array}{c}\text { Mixte } \\
\text { Dominante } \\
\text { inductif }\end{array}$ & $3 / 3$ & $12 / 17$ & $1 / 5$ & $3 / 9$ & justes & $\begin{array}{c}\text { justes } \\
+ \\
\text { abst } \\
+ \\
+ \\
\text { erroné }\end{array}$ & & & justes & justes \\
\hline ESI 6 & $\begin{array}{c}\text { Mixte } \\
\text { Dominante } \\
\text { hypothético-déductif }\end{array}$ & $2 / 3$ & $10 / 17$ & $0 / 5$ & $8 / 9$ & & $\begin{array}{c}\text { Justes } \\
+ \\
\text { abst } \\
\end{array}$ & justes & & justes & justes \\
\hline ESI 7 & Mixte & $1 / 3$ & $14 / 17$ & $0 / 5$ & $4 / 9$ & & justes & & & & justes \\
\hline ESI 8 & $\begin{array}{c}\text { Mixte } \\
\text { Dominante } \\
\text { inductif }\end{array}$ & $2 / 3$ & $11 / 17$ & $2 / 5$ & $7 / 9$ & justes & justes & justes & justes & justes & justes \\
\hline ESI 9 & $\begin{array}{c}\text { Mixte } \\
\text { Dominante } \\
\text { inductif }\end{array}$ & $3 / 3$ & $17 / 17$ & $1 / 5$ & $7 / 9$ & justes & $\begin{array}{c}\text { justes } \\
+ \\
\text { erroné } \\
+ \\
\text { abst } \\
\end{array}$ & justes & & justes & $\begin{array}{c}\text { justes } \\
+ \\
\text { erroné }\end{array}$ \\
\hline ESI 10 & $\begin{array}{c}\text { Mixte } \\
\text { Dominante } \\
\text { hypothético-déductif }\end{array}$ & $1 / 3$ & $15 / 17$ & $0 / 5$ & $9 / 9$ & & $\begin{array}{c}\text { Justes } \\
+ \\
\text { abst }\end{array}$ & justes & & justes & justes \\
\hline ESI 11 & $\begin{array}{c}\text { Mixte } \\
\text { Dominante } \\
\text { hypothético-déductif }\end{array}$ & $2 / 3$ & $15 / 17$ & $1 / 5$ & $5 / 9$ & justes & $\begin{array}{c}\text { Justes } \\
+ \\
\text { abst }\end{array}$ & justes & & & justes \\
\hline ESI 12 & $\begin{array}{c}\text { Mixte } \\
\text { Dominante } \\
\text { hypothético-déductif }\end{array}$ & $3 / 3$ & $15 / 17$ & $1 / 5$ & $6 / 9$ & justes & $\begin{array}{c}\text { justes } \\
+ \\
\text { erroné } \\
+ \\
\text { abst }\end{array}$ & abst & justes & justes & $\begin{array}{c}\text { justes } \\
+ \\
+ \\
\text { erroné }\end{array}$ \\
\hline
\end{tabular}

Légende : Les cellules grisées correspondent à l'absence de liens établis du fait de l'absence de l'identification des risques et/ou de réactions humaines.

Réactions Humaines (RH). 
Perception de leur démarche mentale et de la carte conceptuelle par les étudiants Les propos recueillis auprès des 12 étudiants en fin d'entretien mettent en évidence les points suivants :

- Jugement que portent les étudiants sur leur démarche mentale :

Trois quarts des étudiants (9/12) estiment que leur démarche mentale n'est pas ordonnée, organisée. Les propos les plus souvent cités sont les suivants :

- $\quad$ C'est le fouillis » $(3 / 12)$

- $\quad$ ¿ C'est le bazar » $(3 / 12)$

- «C'est brouillon » (3/12)

- «Ça part dans tous les sens » $(2 / 12)$

Trois étudiants ne portent aucun jugement sur leur démarche mentale.

- Habilités métacognitives:

L'ensemble des étudiants sont capables de verbaliser sur leur propre fonctionnement intellectuel quand ils raisonnent. La richesse des propos tenus est variable d'un étudiant à l'autre.

Certains étudiants identifient avoir établis des liens (7/12) : «j'ai fait des liens avec des choses », «je cherche à voir comment les choses peuvent avoir des liens entre elles », «il y a plein de liens qui se font entre les différents problèmes », «toutes les choses elles sont à peu près mises en lien les unes avec les autres », «les choses mises en lien ça prend forme, ça se hiérarchise ».

D'autres perçoivent qu'ils ont tendance à se disperser (3/12) : «quand je réfléchis, il faut que je reste centré sur le problème et faire tous ce qui va avec et pas partir sur autre chose », «je trouve que je pars d'un point après je m'en vais, je vais à un autre point, je fais des allersretours », «j'ai tendance à beaucoup revenir sur d'autres choses ».

Deux étudiants estiment être lents quand ils raisonnent : «mon raisonnement il est lent », «je ne relève pas rapidement les problèmes ».

Les étudiants expriment aussi des points positifs (4/12) et négatifs (3/12) sur leur fonctionnement intellectuel: Points positifs : «J'ai un regard profond de la situation », «je trouve que c'est assez claire, exhaustif », «ça représente assez bien ce que j'ai compris de la situation », «ça me semble assez juste ». Points négatifs : Un étudiant juge qu'il a « encore du chemin à faire », un autre dit «je n'ai pas du tout priorisé les problèmes »; un troisième explique : «quelques petites erreurs, il faut que je fasse attention à bien relever les bonnes informations ».

- Intérêt que les étudiants attribuent à la carte conceptuelle :

La plupart des étudiants (8/12) estiment que la carte conceptuelle a un intérêt.

En effet, ils considèrent que la carte conceptuelle leur permet d'avoir une vision globale de la situation : «ça permet de mieux visualiser»; « on a une vue d'ensemble »; de s'appuyer dessus pour réajuster : "ça permet de rajouter des liens après coup », " on peut faire des flèches et on peut les gommer si on voit que ça ne va pas »; d'analyser de façon plus exhaustive la situation: «en relisant la carte je n'aurais peut-être pas pensé au risque de chute », «ça nous amène à penser à des choses qu'on aurait pas pu identifier sinon », «ça permet de ne rien oublier $\gg$.

Un étudiant pose un regard négatif sur la conception de la carte : «moi je ne dessinerai pas ça comme ça $[\ldots]$ ça ne me génère pas un schéma qui moi m'aide $[. .$.$] moi les traits dans tous le$ sens ça m'éclaircis pas beaucoup [...] j'ai eu du mal à relire le schéma pour voir comment on peut reprendre l'ensemble des problèmes ».

Parmi l'ensemble des étudiants, 3 ne font pas de commentaires sur l'intérêt des cartes. 
- Difficultés de verbalisation :

Nous observons que sur l'ensemble, 2 étudiants sont en difficultés pour s'exprimer à propos de leur démarche mentale. Cela se traduit par des longs silences ou des réponses qui ne sont pas en lien avec la question que nous posons en fin d'entretien. En effet à la question : "quel regard vous posez sur votre démarche mental ? »Les étudiants réalisent une synthèse de la carte : «y a tout ce qui est propre à la maladie, il y a que des problèmes », «j'ai parlé de sa dépression, j'ai parlé de ce qui s'est passé après sa dépression, j'ai parlé pourquoi elle est à l'hôpital».

- Les interrogations que suscite l'exercice chez les étudiants :

Un étudiant s'interroge sur la manière dont se sont organisés ses camarades pour analyser la situation clinique et si chacun raisonne différemment. Un autre se questionne sur la justesse de son analyse : «la question est de savoir si tout ce que j'ai dit est juste ». L'ensemble des étudiants demande s'ils auront un retour sur leur analyse de la situation simulée.

- Parmi les autres remarques évoquées :

Un étudiant dit avoir eu un déclic quand il a exprimé à haute voix le cheminement de sa pensée : "dire les choses à haute voix m'a permis d'identifier plus de choses », « de dire les choses à haute voix, ça va tout seul je réussis à faire les liens ».

\section{DISCUSSION}

La carte conceptuelle comme outil d'évaluation diagnostique

L'objectif principal de ce travail exploratoire était de vérifier qu'une carte conceptuelle réalisée au cours d'entretiens et construite à partir du discours des étudiants en soins infirmiers lorsqu'ils analysent une situation clinique simulée, est en mesure de rendre visibles leurs stratégies et leurs difficultés de raisonnement.

L'étude montre que la carte conceptuelle construite au cours d'un entretien permet d'identifier les stratégies de raisonnement des étudiants ainsi que les difficultés que rencontrent certains étudiants au cours d'un raisonnement clinique. Les étudiants utilisent en général une stratégie de raisonnement analytique mixte avec soit une dominante inductive, soit une dominante hypothético-déductive. Le fait d'observer ce paramètre dément ce que Nendaz, Charlin, Leblanc, Bordage, 2005, [5] affirment, à savoir : «les cliniciens experts se distinguent des cliniciens novices par l'absence d'utilisation du raisonnement hypothético-déductif ». Selon eux les experts emprunteraient plutôt un raisonnement inductif.

Les difficultés de raisonnement identifiées dans cette étude, semblent liées à l'absence de repérage d'informations importantes concernant le patient (signes et symptômes) d'une part et à l'absence d'analyse des données de la situation permettant de déduire les problèmes de santé, le problème social, les risques, les réactions humaines d'autre part. Elles sont aussi observables par l'absence de liens ou l'existence de liens erronés entre différents éléments de la situation.

Ces résultats concordent avec ceux d'une étude menée par Demeester, Eymard, Vanpee, 2012, [21] auprès de sages-femmes en formation initiale. Celle-ci a mis en évidence les causes de leurs difficultés de raisonnement cliniques ; parmi elles, sont évoquées les causes d'origine cognitive avec les lacunes théoriques, le défaut d'organisation des connaissances, l'insuffisance de mise en lien. Ainsi, nous pouvons nous aussi nous interroger sur une origine cognitive des difficultés de raisonnement que rencontrent les étudiants en soins infirmiers. En effet la performance du raisonnement clinique dépend de la qualité de l'organisation des connaissances dans la mémoire à long terme et de la manière dont ces connaissances sont reliées entre elles sous forme de réseaux. De plus, les connaissances antérieures stockées dans la mémoire à long terme permettent lorsqu'elles sont activées de traiter les informations 
externes, de leur donner du sens et d'élaborer une représentation sémantique adaptée à la résolution de problèmes (Tardif, 1997) [9].

$\mathrm{Au}$ regard de ces éléments, nous pouvons considérer que la carte conceptuelle est un outil aidant à l'évaluation diagnostique. Comme le souligne Edmondson citée par Tardif, 2006, [11] «les cartes conceptuelles offrent aux enseignants la possibilité d'avoir un aperçu de ce que les élèves comprennent [...]. Ces cartes permettent également de noter les erreurs, les omissions et les conceptions erronées $[\ldots] »$.

Les cartes conceptuelles nous ont donc permis d'obtenir des données pour porter un jugement sur la validité des connaissances construites par les étudiants.

La carte conceptuelle comme outil contribuant au développement d'habiletés métacognitives

L'objectif secondaire du travail était de montrer que la carte conceptuelle une fois élaborée permet aux étudiants d'identifier leur propre fonctionnement intellectuel. L'analyse de contenu des verbatim obtenus à la suite de l'élaboration des cartes conceptuelles nous a permis de mettre en évidence que les étudiants étaient en mesure de s'exprimer sur leur démarche mentale et donc de développer leur métacognition. En effet, la métacognition est centrée sur le regard que la personne pose sur sa démarche mentale (Lafortune, Jacob, Herbert, 2000) [22], elle fait référence à la connaissance et au contrôle qu'une personne a sur ses stratégies cognitives (Tardif, 1997) [9].

Elle est définie comme «l'ensemble des jugements, des analyses, des régulations, conscientes ou non (mais qu'il importe de rendre explicites, observables et conscientes) effectuées par l'apprenant sur ses propres performances, dans des situations de PRE, PER ou POST performance » (Leclercq, Poumay, sd) [23]. En regard de notre étude, nous pouvons considérer, que les étudiants portent en effet un jugement sur leur démarche mentale en attribuant un degré de satisfaction sur la performance de leur démarche mentale. Nous pouvons également observer qu'ils analysent aussi leur performance dans la mesure où on retrouve dans les verbatim des justifications et des explications au regard de la qualité de leur démarche mentale : «je cherche à voir comment les choses peuvent avoir des liens entre elles ». Pour les étudiants, la carte conceptuelle est également un support qui permet le réajustement : grâce à elle, ils peuvent opérer une activité de contrôle et de régulation. En effet, ils expriment assez bien leur tendance à se disperser et ce qu'ils doivent changer, modifier, corriger. Ils mettent en marche un processus d'autoévaluation de rétroaction interne (Lafortune, Jacob, Herbert, 2000) [22].

Toujours en lien avec les composantes de la métacognition, nous pouvons observer que les étudiants quand ils s'expriment à propos de leur démarche mentale, possèdent des connaissances sur leur façon d'apprendre, sur leur fonctionnement cognitif personnel, sur leurs points forts et leurs points faibles. Par ailleurs, au travers de propos tenus nous pouvons déduire qu'ils seraient en mesure de développer des connaissances métacognitives liées aux conceptions et perceptions qu'ils pourraient élaborer sur d'autres étudiants : " je me demande comment les autres personnes se sont organisées », " est-ce que c'était très différents à chaque fois » et construire ainsi des représentations par comparaison (Lafortune, Jacob, Herbert, 2000) [22].

Nous avons aussi identifié que parmi les étudiants l'un d'entre eux prend conscience des démarches qu'il utilise pour analyser la situation clinique simulée : «dire les choses à haute voix ; ça va tout seul je réussis à faire les liens ».

Parallèlement, nous avons pu observer que parmi les étudiants, 2 sont en difficultés pour s'exprimer sur leur démarche mentale. Ce sont des étudiants qui présentent par ailleurs des difficultés de raisonnement. Poser un regard métacognitif sur son apprentissage est un acte de pensée complexe, puisqu'il s'agit d'un acte d'intériorisation. Ceci sous-tend qu'un tel 
processus ne peut arriver automatiquement et qu'il nécessite un accompagnement (Lafortune, Jacob, Herbert, 2000) [22].

La métacognition est une composante de la psychologie cognitive qui a pris de plus en plus d'importance dans les actions pédagogiques et elle s'avère fondamentale dans la planification et la gestion de l'enseignement et de l'apprentissage (Tardif, 1997) [9].

Au regard de l'ensemble des éléments discutés, la carte conceptuelle apparaît comme un bon moyen pour aider au développement d'habiletés métacognitives des étudiants en soins infirmiers et ainsi favoriser un apprentissage signifiant.

Notons que 9 étudiants sur 12 qualifient leur démarche mentale comme brouillonne lorsqu'ils observent la carte. Ces propos peuvent s'expliquer par le fait que nous avons placé de façon aléatoire sur la feuille, chaque problème, chaque donnée, signe ou symptôme que l'étudiant énonçait pendant l'entretien, puis nous avons établi les liens de sens entre les différents éléments de la situation au fur et à mesure que l'étudiant les explicitait. Donc en fonction de l'emplacement des éléments de la situation, les flèches qui les reliaient pouvaient parfois partir dans tous les sens et renvoyer à une image brouillonne de la carte. La représentation graphique finale ne reflète donc pas forcément la démarche mentale de l'étudiant car nous n'avons probablement pas été totalement fidèles au cheminement qu'aurait emprunté l'étudiant s'il avait lui-même dessiné sa carte conceptuelle.

\section{Perspectives}

L'étude auprès des sages-femmes avait souligné également des facteurs extrinsèques pouvant expliquer les difficultés rencontrées par les étudiants, en particulier : la faiblesse du dispositif pédagogique, les méthodes et techniques pédagogiques inappropriées, le fractionnement des tâches (Demeester, Eymard, Vanpee, 2012) [21]. Ces mêmes facteurs peuvent être questionnés dans notre étude afin d'envisager le réajustement des enseignements et permettre aux étudiants un meilleur apprentissage du raisonnement clinique.

Nous savons que les connaissances sont construites entre autres à partir de savoirs scientifiques et des savoirs stabilisés dans le cadre d'activités formelles d'enseignement et d'apprentissage (Jouquan, 2010) [24].

Les cartes conceptuelles élaborées dans cette étude ont permis d'approcher les représentations cognitives, construites et emmagasinées dans la mémoire à long terme de chaque étudiant. C'est sur ces fondements que le formateur peut opérer une rétroaction cognitive. En effet, c'est grâce à la rétroaction que l'étudiant reçoit sur ses erreurs qu'il acquiert les nombreux détails supplémentaires qui rend sa connaissance opérationnelle (Lafortune, Saint Pierre, 1994) [25]. Ainsi, la conception d'une carte conceptuelle a permis d'observer la stratégie que l'étudiant applique pour analyser une situation clinique simulée. Dès lors que la stratégie devient observable, il est plus facile d'en discuter, d'en saisir les forces et les faiblesses et de la modifier selon les besoins.

C'est pourquoi, il serait pertinent dans un second temps, d'utiliser les cartes conceptuelles élaborées comme support de feed-back en supervision clinique et inciter ainsi les étudiants à une pratique réflexive (Demeester, Vanpee, Marchand, Eymard, 2010) [10].

Dans une perspective cognitiviste et constructiviste, le rôle du formateur est de rendre visible et accessible à l'apprentissage son processus de résolution de problème. Lafortune, Saint Pierre, 1994 [25], et Chamberland, Hivon, 2005 [26], indiquent que l'apprentissage se fait mieux lorsque les étudiants peuvent échanger au sujet de leurs compréhensions et surtout lorsqu'ils peuvent observer des modèles de rôle expert et s'inspirer de leurs actions et de leurs réflexions. En effet, en servant de modèle, le formateur démontre de façon explicite à l'étudiant les étapes essentielles pour mener à bien une tâche (Tardif, 1997) [9]. La technique 
du modelage comme modalité d'enseignement et d'apprentissage du raisonnement clinique, la carte conceptuelle comme support de feed-back paraissent être intéressantes pour permettre aux étudiants de développer cette compétence professionnelle.

Notons que la co-construction des cartes conceptuelles avec les étudiants a pris en moyenne 48 minutes, cette technique demande donc du temps au formateur. Cette contrainte de temps peut constituer un frein à l'utilisation de cette technique.

\section{Les limites de l'étude}

Cette étude comporte quelques limites. En effet, au-delà du nombre limité d'étudiants 12 au total, l'étude a été menée au sein d'une seule structure de formation, ce qui ne permet pas de transférer les résultats obtenus.

Nous avons interprété les liens de type causal ou conditionnel et les liens d'effets prononcés par les étudiants pour déterminer leurs stratégies de raisonnement, il est possible qu'un deuxième chercheur aurait attribué un autre type de raisonnement à certaines unités de sens. Nous avons aussi interprété et catégorisé les verbatim des étudiants, il est possible qu'un autre chercheur aurait défini une autre catégorisation ; ces éléments peuvent constituer une limite en terme de fiabilité et de validité.

Par ailleurs, l'étude du raisonnement clinique est faite à partir d'une situation clinique simulée écrite sans possibilité pour l'étudiant d'observer et/ou d'interroger le patient. Ainsi elle apprécie uniquement ce que certains auteurs nomment «un bas raisonnement clinique », en opposition au « haut raisonnement clinique » (Psiuk, 2012) [4].

La technique de questionnement lors des entretiens pouvait parfois induire les réponses des étudiants, suggérer des liens, notamment lorsqu'il était demandé à l'étudiant à quel problème il pouvait rattacher certains signes par exemple. Nous n'avons pas toujours interrompu l'étudiant pour qu'il puisse clarifier son raisonnement, il y a probablement des étapes du raisonnement qui nous ont échappé. En conséquence, nous ne pouvons pas affirmer que la carte conceptuelle élaborée reproduit intégralement et scrupuleusement le cheminement qu'emprunte un étudiant quand il raisonne.

Une autre limite en lien avec l'entretien mené auprès du groupe d'étudiant est d'ordre affectif. Nous avons pu observer que deux étudiants ont eu une certaine gêne à exprimer à haute voix leur raisonnement et à exprimer ce qu'il pensait de leur démarche mentale en observant la carte. La situation d'entretien direct avec leur référent pédagogique, pouvant être ressentie comme un temps d'évaluation de ses capacités, a pu produire chez eux une gêne limitant leurs capacités d'expressions. De même, le caractère volontaire des étudiants pour participer à l'étude peut aussi être remis en cause.

\section{CONCLUSION}

Cette étude exploratoire révèle qu'une carte conceptuelle réalisée au cours d'un entretien et construite à partir du discours des étudiants en soins infirmiers lorsqu'ils analysent une situation simulée, est en mesure de rendre compte de leurs stratégies de raisonnement clinique et de leurs difficultés de raisonnement. La carte conceptuelle a donc un intérêt pour contribuer à une évaluation diagnostique des apprentissages concernant le raisonnement clinique. De plus, il semblerait qu'elle constitue aussi une aide au développement des habiletés métacognitives chez les étudiants, ce qui parait intéressant à retenir quand on sait que «parmi les variables cognitives et affectives, c'est la métacognition qui influence le plus l'apprentissage » (Leclercq, Poumay, sd [23]. Ces constats conduisent à réfléchir à des réajustements concernant l'enseignement du raisonnement clinique et à mettre en œuvre des stratégies d'aide à l'apprentissage par l'utilisation des cartes conceptuelles. La carte 
conceptuelle pourrait être utilisée dans un second temps comme support de communication et de feed-back avec les étudiants.

Cette étude nous incite donc à renouveler l'expérience auprès d'un plus grand nombre d'étudiants avec une rétroaction immédiate, d'autre part elle nous encourage à initier nos collègues formateurs à cette technique. Enfin, dans une intention de modelage, elle engage le formateur à élaborer une carte conceptuelle à partir d'une situation clinique simulée pour que les étudiants identifient les processus intellectuels qui s'opèrent quand il raisonne.

Conscientes de l'investigation en temps que génère l'utilisation de cet outil, nous partageons néanmoins les propos tenus par Jouquan, 2010, [24]. «quel qu'en soit le support, l'apprentissage en profondeur et signifiant est une tâche cognitive exigeante pour les étudiants et les enseignants. En effet, ces derniers doivent apporter aux étudiants la supervision nécessaire pour qu'ils réussissent ». 


\section{REFERENCES}

[1] Ministère des affaires sociales et de la santé. Profession infirmier, recueil des principaux textes relatifs à la formation préparant au diplôme d'Etat et à l'exercice de la profession : Berger-Levrault ; 31/07/2009.

[2] COUDRAY MA, GAY C. Le défi des compétences. Elsevier Masson ; 2009.

[3] RAYNAL F., RIEUNIER A. Pédagogie : dictionnaire des concepts clés. Apprentissage, formation, psychologie cognitive. $8^{\text {ème }}$ édition : ESF ; 2010.

[4] PSIUK T. L'apprentissage du raisonnement clinique. Concepts fondamentaux. Contexte et processus d'apprentissage. $1^{\text {ére }}$ éd. De Boeck ; 2012.

[5] NENDAZ M, CHARLIN B, LEBLANC V, BORDAGE G. Le raisonnement clinique: données issues de la recherche et implications pour l'enseignement. Pédagogie Médicale, novembre 2005; 6 (4): 235-254.

[6] W.EVA K. Ce que tout enseignant devrait savoir concernant le raisonnement clinique. Pédagogie Médicale, novembre 2005; 6 (4): 225-234.

[7] MASQUELET A-C. Le raisonnement médical. Presses universitaires de France; 2006.

[8] AUDÉTAT MC, LAURIN S, SANCHE G. Aborder le raisonnement clinique du point de vue pédagogique. I. Un cadre conceptuel pour identifier les problèmes de raisonnement clinique. Pédagogie Médicale, novembre $2011 ; 12$ : 223-229.

[9] TARDIF J. Pour un enseignement stratégique. L'apport de la psychologie cognitive. Les éditions logiques ; 1997.

[10] DEMEESTER A; VANPEE D; MARCHAND C; EYMARD C. Formation au raisonnement Clinique: perspectives d'utilisation des cartes conceptuelles. Pédagogie Médicale, mai $2010 ; 11: 81-95$.

[11] TARDIF J. L'évaluation des compétences. Documenter le parcours de développement. Montréal : Chenelière Education ; 2006.

[12] MARCHAND C, IVERNOIS (D') JF. Les cartes conceptuelles dans les formations en santé. Pédagogie Médicale, novembre 2004 ; 5 : 230-240.

[13] NOVAK JD, CANAS AJ. The Theory Underlying Concept Maps and How to Construct and Use Them Institute for Human and Machine Cognition.

Technical Report IHMC CmapTools 2006-01 Rev 01.2008. http://cmap.ihmc.us/Publications/ResearchPapers/TheoryCmaps/TheoryUnderlyingConceptM aps.htm. Consulté le 09/06/2013

[14] Daley BJ, Torre DM. Concept maps in medical education: an analytical literature review. Medical Education, mai $2010 ; 44: 440-8$. 
[15] MARCHAND C, GAGNAYRE R. Utilisation de la carte conceptuelle auprès de formateurs en santé pour l'apprentissage de concepts pédagogiques. Pédagogie Médicale, février $2004 ; 5: 13-23$.

[16] POTTIER P, PLANCHON B, HARDOUIN JB, SEBILLE V, ROGEZ JM, BARRIER JH. Evaluation préliminaire de la validité de construit d'un dispositif cartographique (carte procédurale) pour l'étude du raisonnement clinique. Pédagogie Médicale, mai 2011; 12: 87101.

[17] MARCHAND C, D'IVERNOIS JF, ASSAL JP, SLAMA G, HIVON R. An analysis, using concept mapping, of diabetic patients' knowledge, before and after patient education. Medical Teacher, janvier 2002; 24 :90-9.

[18] VERMERSCH P. L'entretien d'explicitation. $3^{\mathrm{e}}$ édition : Editions ESF, 2000.

[19] PHANEUF M. La réflexion à voix haute, complément ou substitut de la démarche de soins. 2009, [].: www.infiressources.ca/.../La_reflexion_a_voix_haute_complement_o. consulté le 16/03/2013

[20] FORTIN MF. Fondements et étapes du processus de recherche. Méthodes quantitatives et qualitatives. $2^{\mathrm{e}}$ édition, Chenelière Education, 2010.

[21] DEMEESTER A, EYMARD C, VANPEE D. Apprentissage du raisonnement clinique : difficultés identifiées en formation initiale sage-femme. Revue française de pédagogie. octnov-déc 2012; $181: 43-54$.

[22] LAFORTUNE L., JACOB S., HERBERT D. Pour guider la métacognition. Presse Universitaire du Québec ; 2000.

[23] LECLERCQ D, POUMAY M. Une définition opérationnelle de la métacognition et ses mises en œuvre. LabSET - Université de Liège, s.d., 40 pages. http://www.netvibes.com/mariannepoumay. Consulté le 25/03/2013

[24] JOUQUAN J. S'il te plaît,...dessine-moi tes connaissances ! Pédagogie Médicale, mai $2010 ; 11: 77-79$.

[25] LAFORTUNE L, SAINT PIERRE L. Les processus mentaux et les émotions dans l'apprentissage. Logiques ; 1994.

[26] CHAMBERLAND M, HIVON R. Les compétences de l'enseignant clinicien et le modèle de rôle en formation clinique. Pédagogie Médicale, mai 2005 ; 6 : 98 - 111. 


\section{ANNEXE I}

\section{Situation clinique simulée}

Madame Valet, âgée de 40 ans, est entrée le 18 avril 2013 en psychiatrie pour névrose hystérique en phase dépressive. Elle revient ce jour, 20 avril 2013, dans ce même service après un séjour de $24 \mathrm{~h}$ en chirurgie orthopédique pour traitement d'une fracture de Pouteau-Colles consécutive à une chute.

Vous êtes infirmier(e) dans le service de psychiatrie, vous accueillez cette patiente ce matin à $9 \mathrm{~h}$ et vous prenez connaissance de l'histoire de la personne et de sa pathologie.

Histoire de la personne et de sa pathologie :

Madame Valet habite depuis 7 ans dans un petit village en banlieue parisienne avec ses 3 enfants. Auparavant, elle résidait dans la région de Lille avec son concubin, père de ses 3 enfants. Elle l'a quitté, car il s'alcoolisait à longueur de journée et l'a laissée sans argent. Depuis, son concubin ne s'est jamais intéressé à ses enfants mais malgré tout Madame Valet a toujours peur qu'il veuille les lui reprendre. Elle a donc ses 3 enfants à charge, son concubin n'ayant aucun droit :

- Sa fille aînée, âgée de 17 ans est en première ES. Elle est souvent en conflit avec sa mère entraînant parfois des disputes violentes.

- $\quad$ La cadette, âgée de 12 ans, fréquente un collège pour enfants en difficulté. Elle est interne et rentre toutes les fins de semaine.

- $\quad$ Le dernier, un garçon âgé de 7 ans, porte le même prénom que le père de la patiente. Madame Valet dit : « c'est mon petit chéri !».

Sa biographie est difficile à établir, la patiente ayant des difficultés à évoquer certains souvenirs anciens tout en étant capable d'en évoquer d'autres.

Elle affirme avoir été mal aimée par ses parents. Elle a son Bac et un BTS de secrétariat.

Elle a exercé un travail de secrétaire pendant 3 ans mais avec difficultés car elle était souvent absente. A cette époque, elle présentait des céphalées violentes. Par la suite, elle a préféré s'occuper de ses enfants.

En mars 2009, elle a été admise dans le service de cardiologie de l'hôpital à la suite de la survenue de précordialgies aiguës le jour même où elle apprenait le placement de sa seconde fille décidé par le juge des enfants.

Elle avait la sensation que «son cœur allait exploser». Le bilan cardiaque ne révélait aucune anomalie particulière ; elle rencontrait alors un psychiatre: il a été décidé son transfert dans l'unité psychiatrique où elle restera hospitalisée une semaine.

Un traitement anxiolytique et une prise en charge psycho-thérapeutique pendant 3 ans avaient amélioré son état psychique. Quelques semaines après sa sortie, sa petite sœur Annie, âgée de 22 ans, se jette d'un pont, elle restera paraplégique. La patiente se culpabilise de ne pouvoir l'aider puisqu'elle se trouve à plus de $900 \mathrm{~km}$.

Sur le plan sociale, elle bénéficie d'une aide-ménagère et d'une allocation aux adultes handicapés pour raisons psychiatriques et ceci depuis 2012, malgré l'avis défavorable de son psychiatre ; mais dit-elle, «j'ai alors changé de psychiatre et mon médecin traitant m'a aidé à obtenir la pension ». Depuis cette période, elle n'a plus de traitement.

Hospitalisation actuelle :

Elle se présente aux urgences de l'hôpital le 18 avril 2013 à 14h. Devant son état, il est décidé son transfert dans l'unité de psychiatrie, la patiente acceptant les soins.

Le diagnostic retenu par le psychiatre est le suivant : névrose hystérique en phase dépressive.

Le matin du 19 avril 2013, on la retrouve allongée sur le sol se plaignant du poignet droit. Lorsqu'on essaie de la lever, elle dit avoir la tête qui tourne et reste dans l'impossibilité de maintenir la station debout.

Après bilan radiologique, le diagnostic de fracture de Pouteau-Colles déplacée est posé. Madame Valet est transférée en chirurgie orthopédique. Le 19 avril 2013 matin, il est réalisé une ostéosynthèse par 3 broches transcutanées sous anesthésie loco-régionale. Une attelle plâtrée a été mise en place au membre supérieur droit et un traitement antalgique a été prescrit. Madame Valet revient en psychiatrie le 20 avril 2013 à $9 \mathrm{~h}$ avec un plâtre circulaire en résine, l'avant-bras droit est soutenu par une écharpe, elle est en fauteuil roulant.

La prescription médicale indique :

- $\quad$ Anafranil ${ }^{\circledR} 75$ (Clomipramine anti-dépresseur tricyclique) : $1 \mathrm{cp}$ le soir

- $\quad$ Lexomil@ (Benzodiazépine anxiolytique) $: 1 \mathrm{cp} / \mathrm{j}$ soit $1 / 4 \mathrm{cp}$ le matin, $1 / 4 \mathrm{cp}$ le midi, $1 / 2 \mathrm{cp}$ le soir.

- $\quad$ Imovane ${ }^{\circledR}$ (hypnotique non barbiturique) : $1 \mathrm{cp}$ au coucher

- Radio avant-bras droit et profil

- Perfalgan ${ }^{\circledR}$ (antalgique) 1 gr à passer sur 15 min, à renouveler toutes les $6 \mathrm{~h}$ pendant $48 \mathrm{~h}$, à partir de $10 \mathrm{~h}$

- Prise des paramètres vitaux 3 fois par jour (Pouls, tension artérielle, température)

Vous la prenez en charge à ce moment-là. Elle est installée en chambre seule à sa demande. 
Vous réalisez le recueil de données suivant :

\begin{tabular}{|c|c|c|}
\hline BESOINS & $\begin{array}{l}\text { Manifestations } \\
\text { d'indépendance }\end{array}$ & Manifestations de dépendance \\
\hline Respirer & $\begin{array}{l}\text { Fréquence respiratoire } \\
\text { normale }\end{array}$ & \\
\hline Dormir et se reposer & & $\begin{array}{l}\text { Dit ne plus dormir depuis une semaine. Dit } \\
\text { s'endormir } 2 \mathrm{~h} \text { après s'être couchée avec } \\
\text { plusieurs réveils dans la nuit. Dit faire des } \\
\text { cauchemars. } \\
\text { Elle a des cernes sous les yeux. } \\
\text { Dit se sentir très fatiguée. }\end{array}$ \\
\hline Boire et manger & & $\begin{array}{l}\text { Dit s'alimenter qu'avec des liquides. N'a } \\
\text { accepté qu'un café au petit déjeuner. }\end{array}$ \\
\hline Eliminer & $\begin{array}{l}\text { Dit avoir une selle par } \\
\text { jour. }\end{array}$ & \\
\hline $\begin{array}{l}\text { Etre propre, soigner et } \\
\text { protéger ses téguments }\end{array}$ & & $\begin{array}{l}\text { Ne peut pas se laver seule } \\
\text { Dit ne pas avoir envie de se laver }\end{array}$ \\
\hline Se vêtir et se dévêtir & $\begin{array}{l}\text { A en sa possession des } \\
\text { vêtements personnels }\end{array}$ & $\begin{array}{l}\text { Elle a peur que ses vêtements ne soient pas } \\
\text { adaptés au plâtre }\end{array}$ \\
\hline $\begin{array}{l}\text { Se mouvoir et maintenir } \\
\text { une bonne posture }\end{array}$ & & $\begin{array}{l}\text { Elle dit avoir des difficultés à s'adapter au } \\
\text { plâtre } \\
\text { Dit avoir le cœur qui bat trop vite. } \\
\text { Se montre incapable de se mettre debout, et } \\
\text { de se servir correctement de ses mains. }\end{array}$ \\
\hline $\begin{array}{l}\text { Maintenir la température } \\
\text { dans les limites de la } \\
\text { normale }\end{array}$ & $\mathrm{T}^{\circ}: 37^{\circ} \mathrm{C}$ & \\
\hline $\begin{array}{l}\text { Communiquer avec ses } \\
\text { semblables }\end{array}$ & & $\begin{array}{l}\text { Pendant l'entretien, ses propos sont réduits, } \\
\text { débit lent, paupières baissées. Son visage } \\
\text { exprime de la lassitude. } \\
\text { Se dit inquiète de savoir ses enfants en } \\
\text { famille d'accueil pendant son } \\
\text { hospitalisation. } \\
\text { Dit se sentir seule. }\end{array}$ \\
\hline Eviter les dangers & $\begin{array}{l}\text { Ne présente pas de } \\
\text { déficit sensoriel } \\
\text { Elle mobilise } \\
\text { normalement sa main } \\
\text { droite }\end{array}$ & $\begin{array}{l}\text { Elle porte un plâtre au niveau de l'avant } \\
\text { bras droit. } \\
\text { Au sujet de sa fracture elle dit que c'est la } \\
\text { goutte d'eau qui fait déborder le vase et } \\
\text { qu'il vaudrait mieux en finir. } \\
\text { Elle se plaint de douleurs diffuses. } \\
\text { A des vertiges lorsqu'elle se met debout. } \\
\text { Elle pèse 50kg pour } 1 \mathrm{~m} 70 \text {. }\end{array}$ \\
\hline Se recréer & & Dit ne pas avoir le cœur à se distraire. \\
\hline $\begin{array}{l}\text { S'occuper en vue de se } \\
\text { réaliser }\end{array}$ & & $\begin{array}{l}\text { Elle affirme être incapable d'élever ses } \\
\text { enfants. }\end{array}$ \\
\hline
\end{tabular}


ANNEXE II

Grille de référence

\begin{tabular}{|c|c|c|}
\hline Problèmes réels & $\begin{array}{c}\text { Risques de problèmes } \\
\text { médicaux et de réactions } \\
\text { humaines physiques et } \\
\text { psychologiques }\end{array}$ & $\begin{array}{c}\text { Réactions humaines } \\
\text { physiques et psychologiques } \\
\text { réelles }\end{array}$ \\
\hline $\begin{array}{l}\text { Fracture du Pouteau- } \\
\text { Colles liée à une chute se } \\
\text { manifestant par une douleur } \\
\text { au poignet droit et } \\
\text { confirmée par la radio. }\end{array}$ & $\begin{array}{l}\text { Risque de syndrome de } \\
\text { Volkmann lié à la fracture du } \\
\text { Pouteau-Colles. }\end{array}$ & $\begin{array}{l}\text { Altération de la mobilité } \\
\text { physique lié au plâtre au } \\
\text { niveau de l'avant-bras droit, à } \\
\text { la douleur, à la dépression, se } \\
\text { manifestant par une incapacité } \\
\text { à se mettre debout et se servir } \\
\text { correctement de ses mains, } \\
\text { elle a des vertiges. }\end{array}$ \\
\hline $\begin{array}{l}\text { Névrose hystérique en } \\
\text { phase dépressive se } \\
\text { manifestant par des propos } \\
\text { réduit, un débit lent, des } \\
\text { paupières baissées. Son } \\
\text { visage exprime de la } \\
\text { lassitude. Dit ne pas avoir le } \\
\text { cœur à se distraire. }\end{array}$ & $\begin{array}{l}\text { Risque infectieux lié à } \\
\text { l'intervention chirurgicale } \\
\text { (ostéosynthése par } 3 \text { broches } \\
\text { transcutanées). }\end{array}$ & $\begin{array}{l}\text { Incapacité de s'adapter à un } \\
\text { changement dans l'état de } \\
\text { santé lié à une invalidité } \\
\text { temporaire (présence d'un } \\
\text { plâtre circulaire en résine à } \\
\text { l'avant-bras droit) se } \\
\text { manifestant par: dit avoir des } \\
\text { difficultés à s'adapter au } \\
\text { plâtre, a peur que ses } \\
\text { vêtements ne soient pas } \\
\text { adaptés au plâtre. }\end{array}$ \\
\hline \multirow[t]{3}{*}{$\begin{array}{l}\text { Problème social lié à la } \\
\text { dépression et le manque et } \\
\text { la perte de ressources } \\
\text { personnelle. }\end{array}$} & $\begin{array}{l}\text { Risque de chute liée au fait } \\
\text { que la patiente a des vertiges } \\
\text { lorsqu'elle se met debout. }\end{array}$ & $\begin{array}{l}\text { Douleur liée à la fracture, à la } \\
\text { dépression se manifestant par } \\
\text { une plainte de douleurs } \\
\text { diffuses. }\end{array}$ \\
\hline & $\begin{array}{l}\text { Risque de suicide lié au fait } \\
\text { que la patiente tient des } \\
\text { propos suicidaire. }\end{array}$ & $\begin{array}{l}\text { Anxiété majeure liée au } \\
\text { contexte familiale difficile, } \\
\text { l'hospitalisation, la fracture se } \\
\text { manifestant par un sommeil } \\
\text { perturbé, des palpitations, } \\
\text { l'inquiétude de savoir ses } \\
\text { enfants en famille d'accueil. }\end{array}$ \\
\hline & $\begin{array}{l}\text { Risque de dénutrition liée au } \\
\text { fait que la patiente s'alimente } \\
\text { peu. }\end{array}$ & $\begin{array}{l}\text { Incapacité à effectuer ses } \\
\text { soins d'hygiène et de } \\
\text { confort, à se vêtir liée à la } \\
\text { fracture, la dépression, se } \\
\text { manifestant par le fait que la } \\
\text { patiente dit ne pas pouvoir se } \\
\text { laver seule, ne pas avoir envie } \\
\text { de se laver, peur que ses } \\
\text { vêtement ne soient pas adaptés } \\
\text { au plâtre. }\end{array}$ \\
\hline
\end{tabular}




\begin{tabular}{|l|l|l|}
\hline Problèmes réels & $\begin{array}{c}\text { Risques de problèmes } \\
\text { médicaux et de réactions } \\
\text { humaines physiques et } \\
\text { psychologiques }\end{array}$ & $\begin{array}{c}\text { Réactions humaines } \\
\text { physiques et psychologiques } \\
\text { réelles }\end{array}$ \\
\hline & $\begin{array}{l}\text { Altération des habitudes de } \\
\text { sommeil liée à la dépression, } \\
\text { l'anxiété, contexte } \\
\text { psychosocial, douleurs } \\
\text { diffuses se manifestant par : } \\
\text { dit ne plus dormir, s'endort 2h } \\
\text { après s'être couchée avec } \\
\text { plusieurs réveils dans la nuit, a } \\
\text { des cernes sous les yeux. }\end{array}$ \\
\hline & $\begin{array}{l}\text { Fatigue liée à la dépression se } \\
\text { manifestant par : dit se sentir } \\
\text { fatiguée }\end{array}$ \\
\hline & $\begin{array}{l}\text { Perturbation dans l'exercice } \\
\text { du rôle parental lié au fait } \\
\text { que les enfants soient placés } \\
\text { en famille d'accueil se } \\
\text { manifestant par : la patiente } \\
\text { affirme âtre incapable d'élever } \\
\text { ses enfants. }\end{array}$ \\
\hline & $\begin{array}{l}\text { Isolement lié à la dépression, } \\
\text { au contexte psychosocial se } \\
\text { manifestant par: dit se sentir } \\
\text { seule }\end{array}$ \\
\hline &
\end{tabular}


ANNEXE III

\section{Cartes conceptuelles}
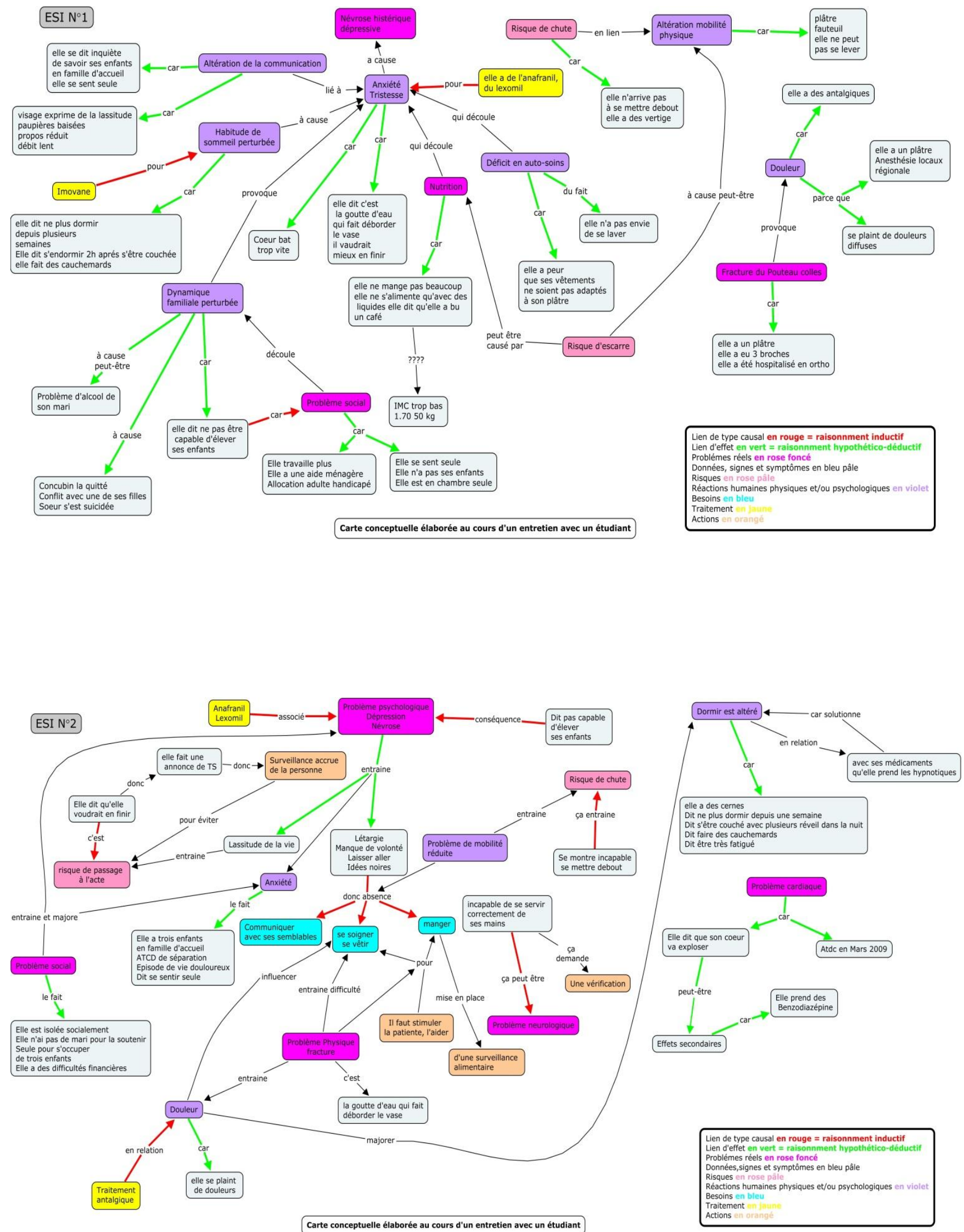
ESI N ${ }^{\circ}$

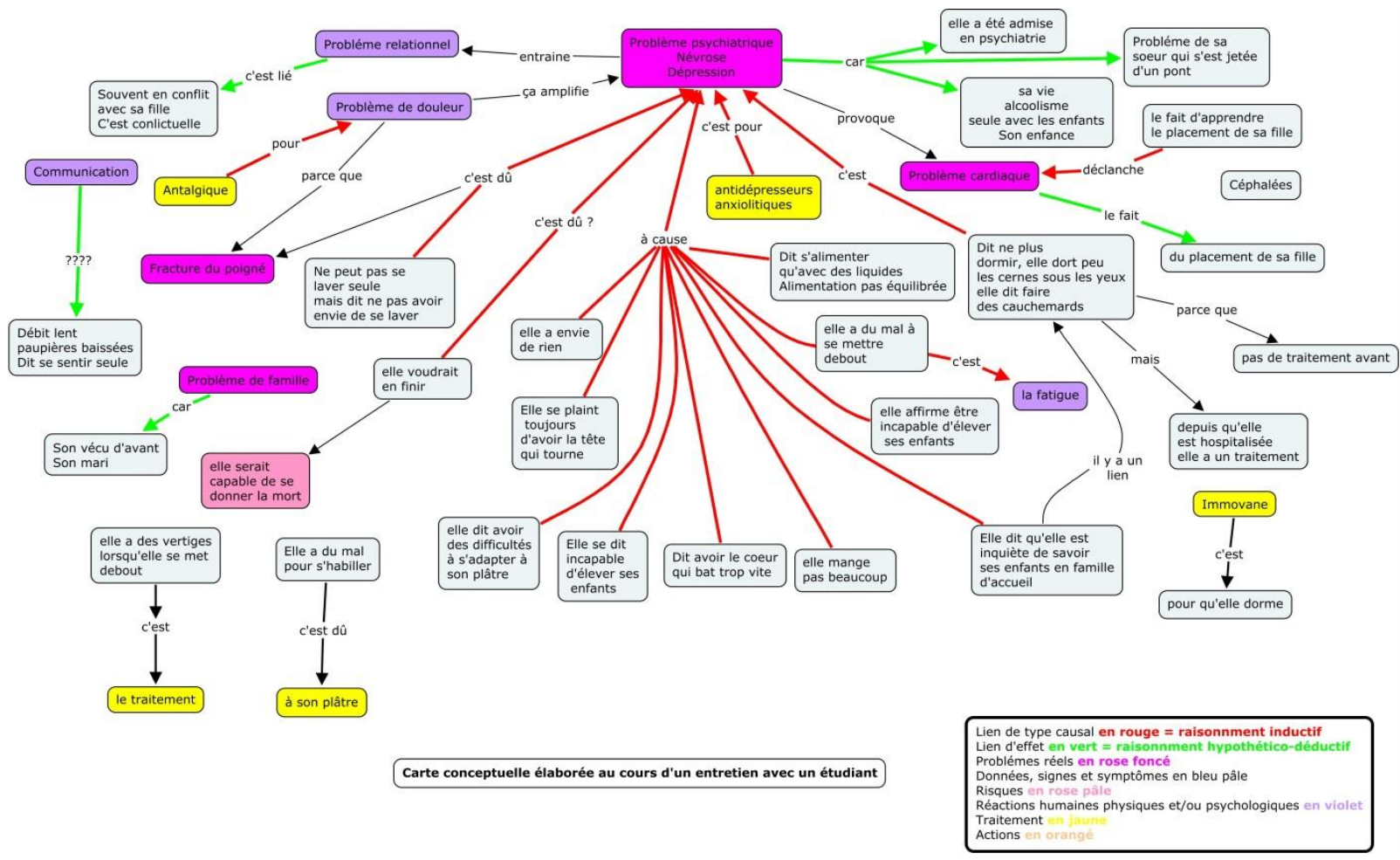

ESI N 04

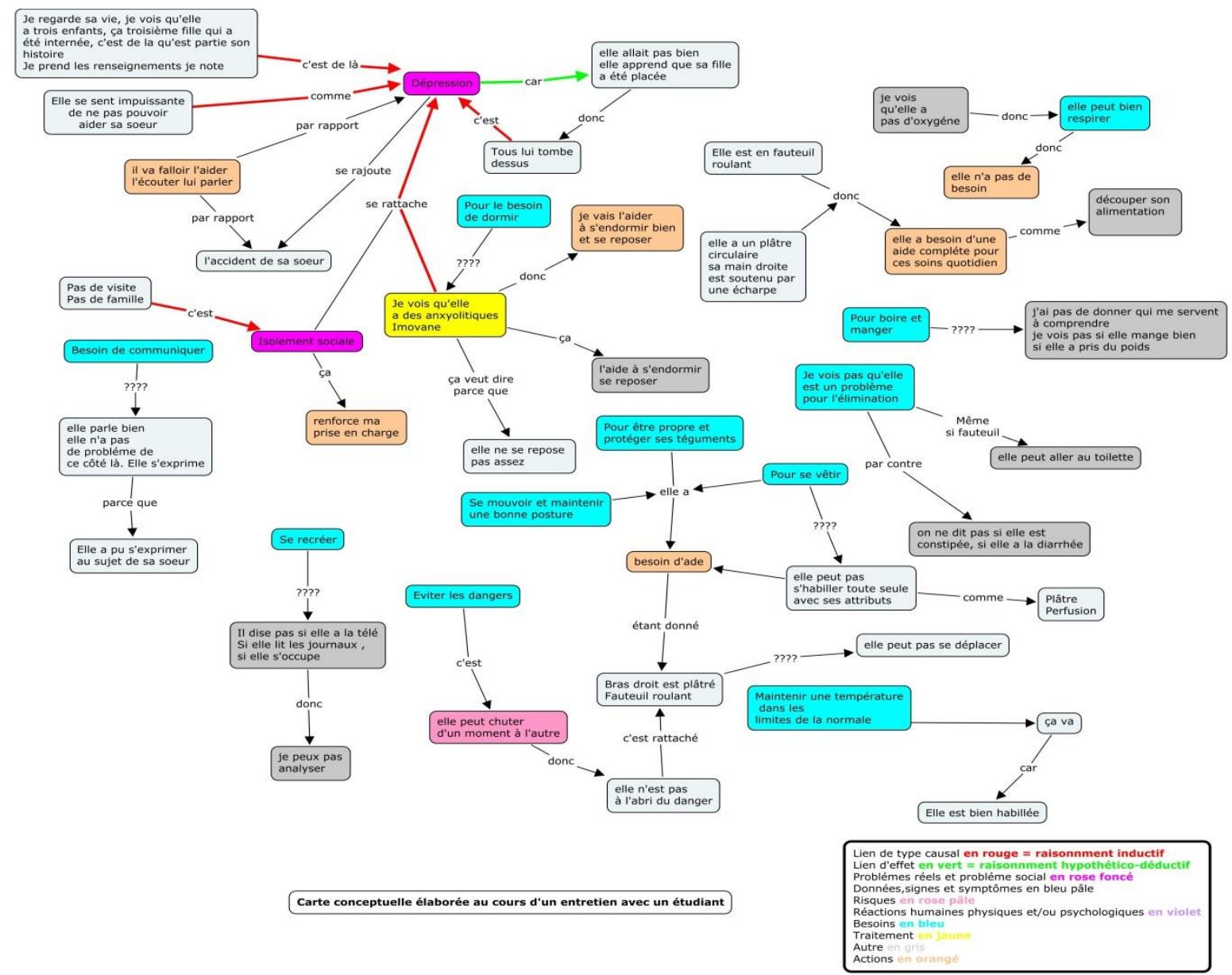




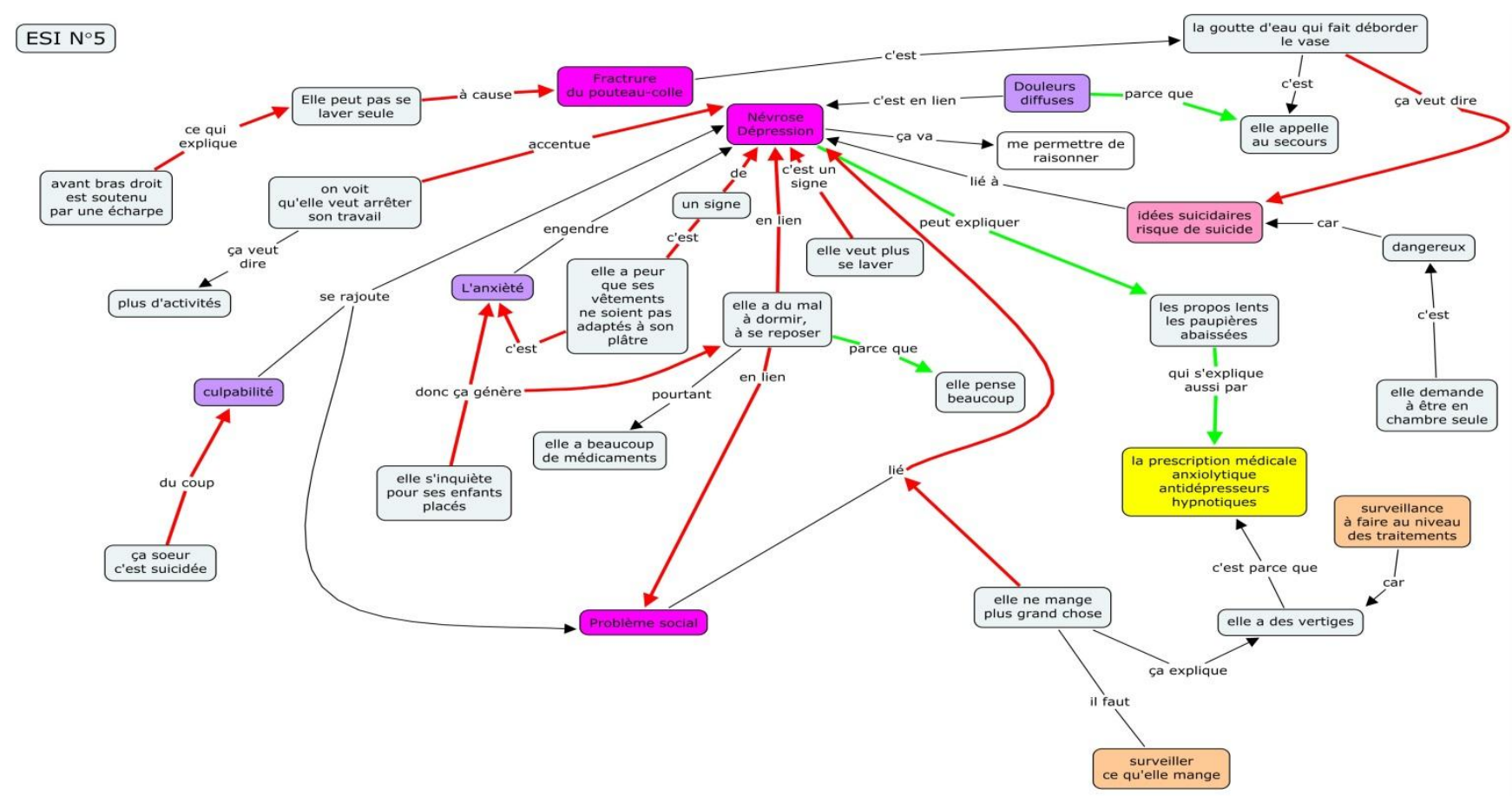

Carte conceptuelle élaborée au cours d'un entretien avec un étudiant

Lien de type causal en rouge = raisonnment inductif
Lien d'effet en vert = raisonnment hypothético-déductif
Problémes réels en rose foncé
Données, signes et symptômes en bleu pâle
Risqueses en rose pale
Réactions humaines physiques et/ou psychologiques en violet
Besoins en bleu
Traitement
Actions on

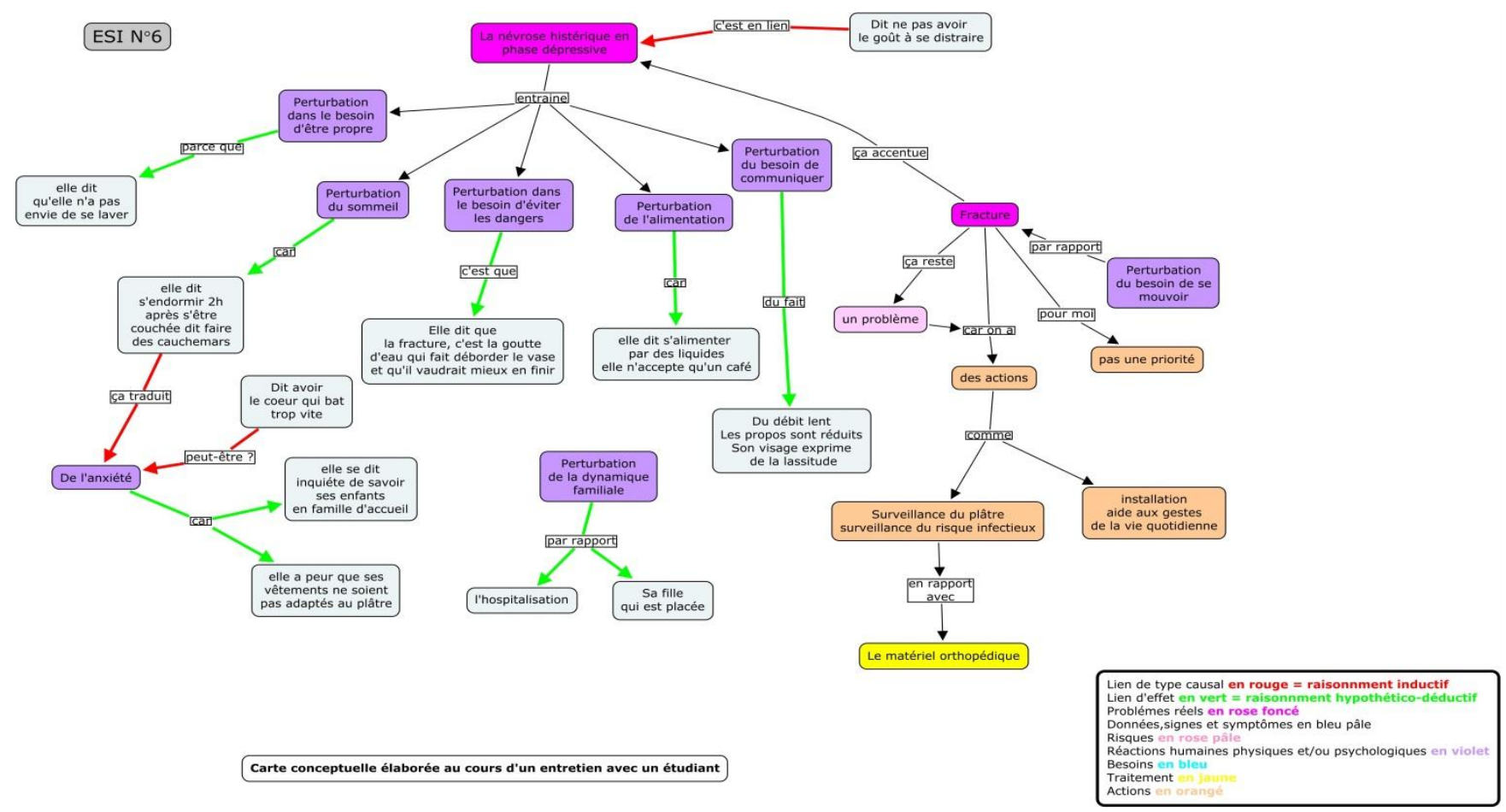



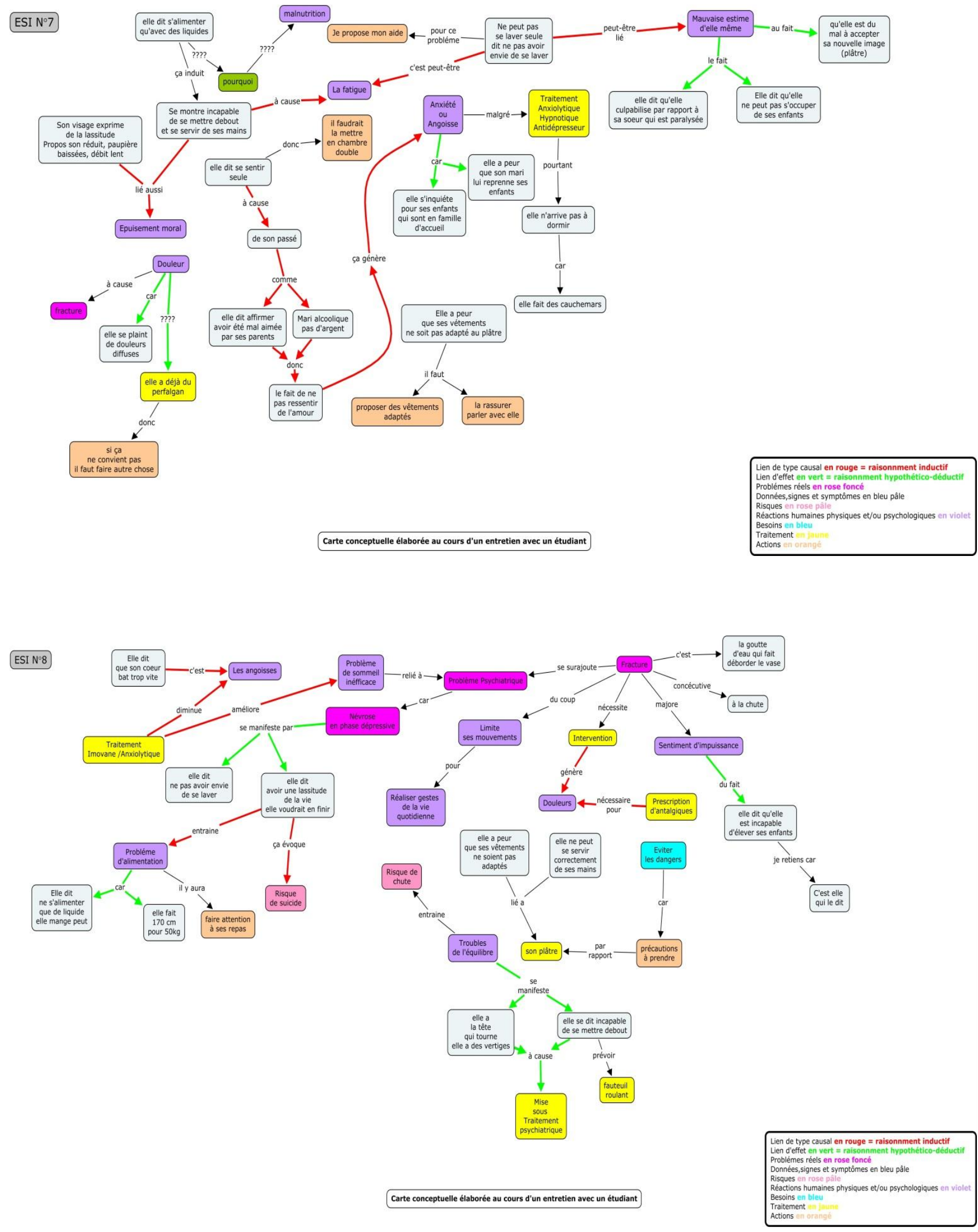

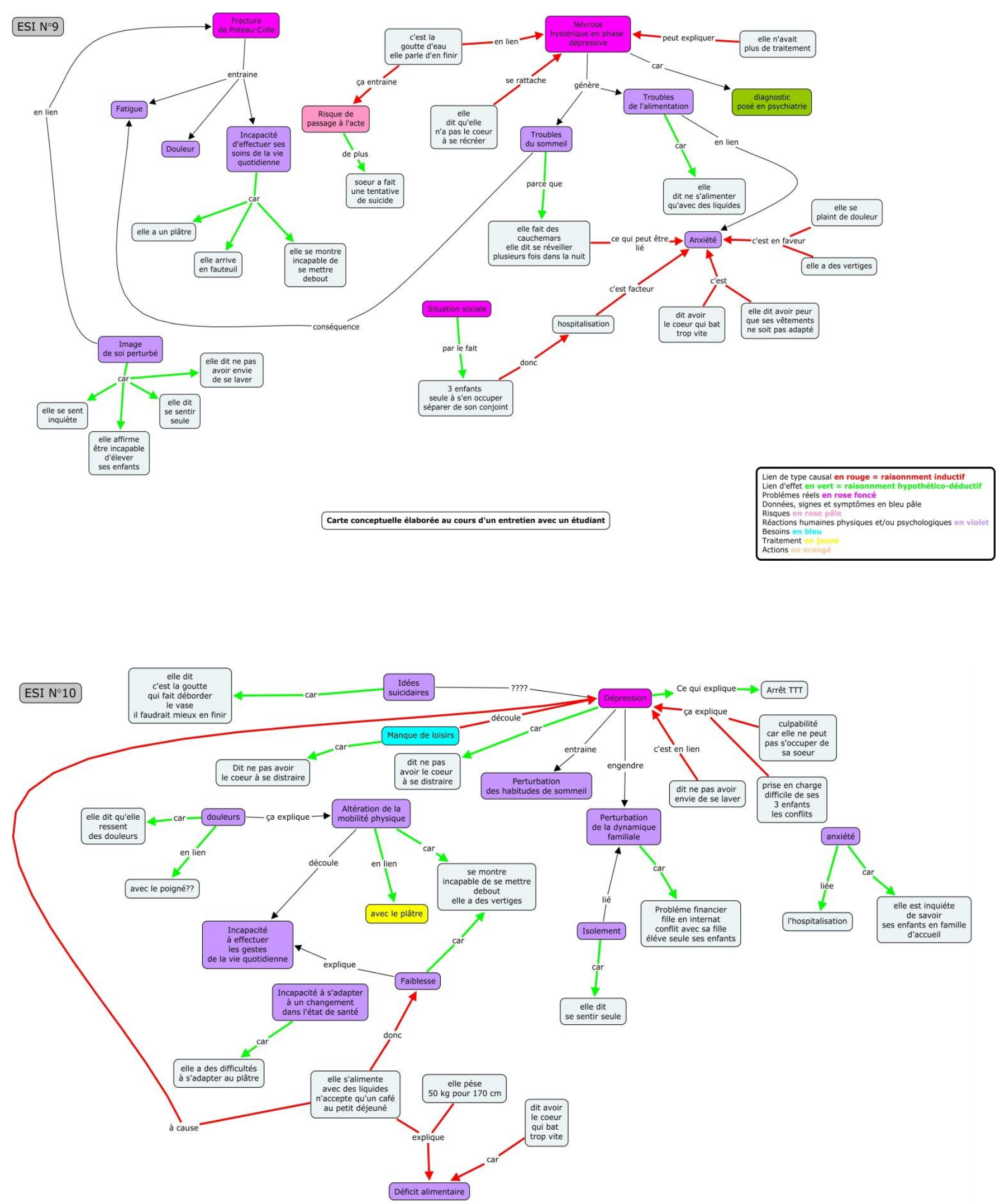

Carte conceptuelle élaborée au cours d'un entretien avec un étudiant

Lien de type causal en rouge $=$ raisonnment inductif
Lien d'effete en vert $=$ raisonnment hypothético-déductif
Problémes réels en rose foncé Problémes réels en rose foncé
Données, signes et symptômes en bleu pâle Risques en rose pale Réactions humaines physiques et/ou psychologiques en violet Besoins en
Traitement 

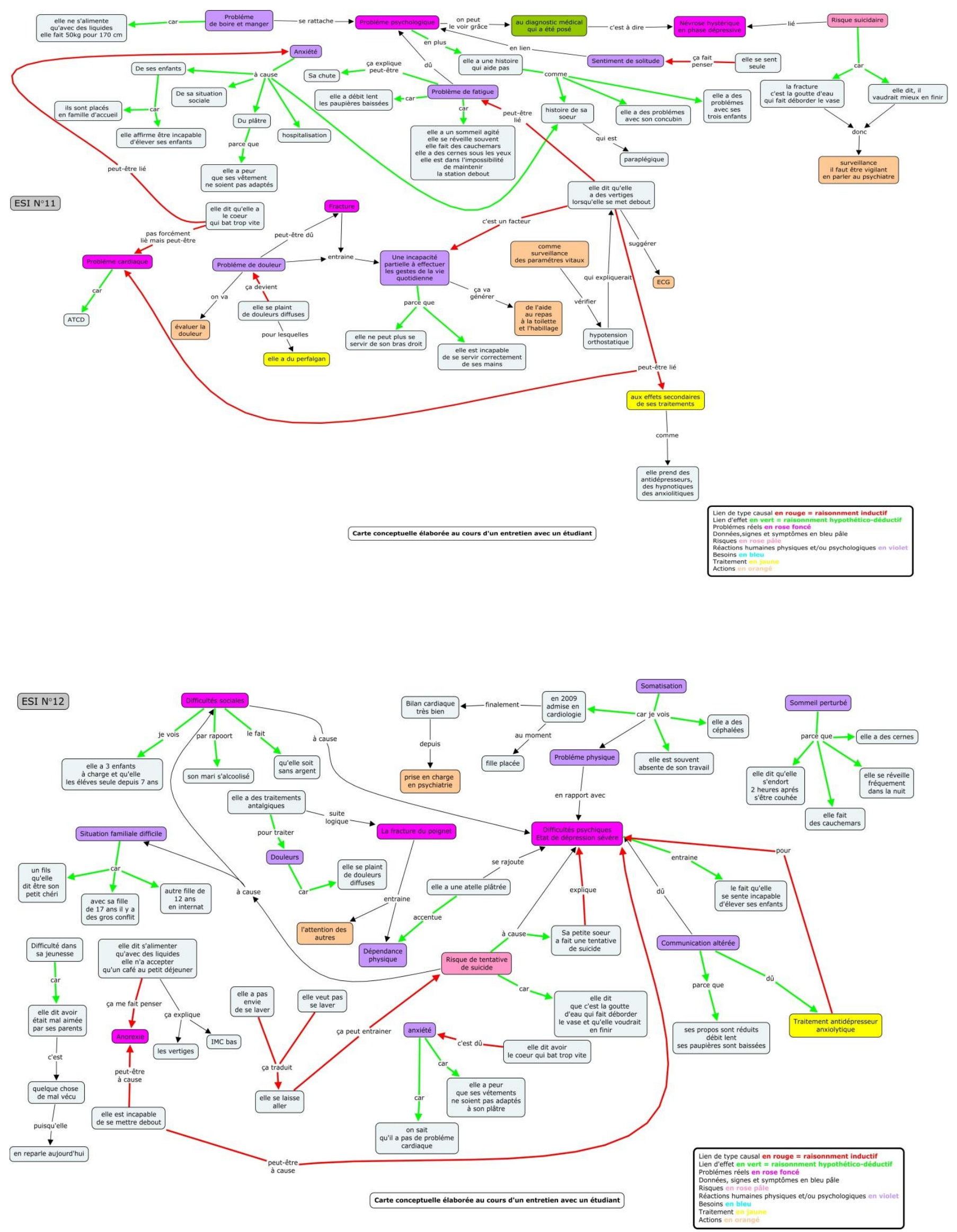